\title{
Biophysical characterization of two commercially available preparations of the drug containing Escherichia coli L-Asparaginase 2
}

Talita Stelling de Araújo ${ }^{1,2,3}$, Sandra M. N. Scapin ${ }^{4}$, William de Andrade ${ }^{1}$, Maira Fasciotti $^{4}$, Mariana T. Q. de Magalhães ${ }^{5}$, Marcius S. Almeida ${ }^{\star 2,3}$, Luís Maurício T.

${ }^{1}$ Laboratory for Pharmaceutical Biotechnology - pbiotech, Faculty of Pharmacy, Federal University of Rio de Janeiro, Rio de Janeiro, RJ, 21941-902, Brazil.

${ }^{2}$ Protein Advanced Biochemistry - PAB, National Center for Structural Biology and Bioimaging - CENABIO, Federal University of Rio de Janeiro, Rio de Janeiro, RJ, 21941-902, Brazil.

${ }^{3}$ Institute for Medical Biochemistry Leopoldo DeMeis, Federal University of Rio de Janeiro, Rio de Janeiro, RJ, 21941-902, Brazil.

${ }^{4}$ National Institute of Metrology, Quality and Technology - INMETRO, Duque de

${ }^{5}$ Department of Biochemistry and Immunology, Federal University of Minas Gerais, Belo Horizonte, Minas Gerais 31270-901, Brazil

*Both authors share senior authorship

\section{Corresponding Author:}

$$
\begin{aligned}
& \text { Luís Maurício T. R. Lima } \\
& \text { E-mail: Mauricio@pharma.ufrj.br } \\
& \text { Marcius da Silva Almeida } \\
& \text { E-mail: msalmeida@cenabio.ufrj.br }
\end{aligned}
$$

Keywords: L-Asparaginase, ligand-binding protein, protein conformation, protein crystallization, protein dynamic, enzyme mechanism, cancer therapy, leukemia, nuclear magnetic resonance (NMR), biopharmaceuticals 


\section{Abstract}

The hydrolysis of asparagine and glutamine by L-asparaginase has been used to treat acute lymphoblastic leukemia for over four decades. Each L-asparaginase monomer has a long loop that closes over the active site upon substrate binding, acting as a lid. Here we present a comparative study two commercially available preparations of the drug containing Escherichia coli L-Asparaginase 2, performed by a comprehensive array of biophysical and biochemical approaches. We report the oligomeric landscape and conformational and dynamic plasticity of $E$. coli type $2 \mathrm{~L}$-asparaginase (EcA2) present in two different formulations, and its relationship with L-aspartic acid, which is present in Aginasa, but not in Leuginase. EcA2 shows a composition of monomers and oligomers up to tetramers, which is mostly not altered in the presence of L-Asp. The N-terminal loop of Leuginase, which is part of the active site is flexibly disordered, but gets ordered as in Aginasa in the presence os L-Asp, while L-Glu only does so to a limited extent. Ion-mobility spectrometry-mass spectrometry reveals two conformers for the monomeric EcA2, one of which can selectively bind to L-Asp and L-Glu. Aginasa has higher resistance to in vitro proteolysis than Leuginase, and this is directly related to the presence of L-Asp. 


\section{Introduction}

L-Asparaginase is an amidohydrolase found in a variety of microorganisms, plants, and animals. It catalyzes the hydrolysis of L-asparagine (L-Asn) to L-aspartate (LAsp) and ammonia. The enzyme is widely used for the treatment of several hematopoietic malignancies and is a crucial element in the treatment of acute lymphoblastic leukemia (ALL) in children, with increasing use in adult chemotherapy [1]. The L-asparaginase mechanism of action consists of serum LAsn depletion since, unlike normal cells, leukemic cells are incapable of synthesizing this amino acid, leading to protein synthesis deficiency and cell death [2]. Most isoforms of L-asparaginases exhibit an L-glutaminase secondary activity, which has been demonstrated to be essential for full anti-leukemic efficacy $(3,4)$. Despite its clinical relevance, several important toxic side effects attributed to serum L-glutamine (L-Gln) depletion have been observed in patients receiving Lasparaginase therapy, including immunosuppression, hepatotoxicity, encephalopathy, pancreatitis, and coagulation dysfunction. Therefore, engineering or identifying enzymes that retain asparaginase and reduced glutaminase activity has been claimed to help decrease toxic effects [5], which requires a detailed understanding of the structural basis involved in substrate recognition by Lasparaginases.

The crystal structure of asparaginase was first reported in 1993 for enzymes from ErA [6] and EcA2 [7], both bound to L-Asp. The active form of all bacterial Lasparaginases is described as homotetramers of about 130 to $140 \mathrm{kDa}$, usually referred to as a dimer of dimers, with four identical non-cooperative active sites formed at the intimate dimer interface. Asparaginase topology is well-conserved among the microorganisms and consists of two domains characterized by an $\alpha / \beta$ fold [8]. The N-terminal domain contains the catalytic site that, when occupied by the substrate, modulates the closure of a 20-amino acid flexible loop (residues 10 to 35 on mature EcA2), which acts as a lid over the active site. The structural integrity of this element is essential for asparaginase activity since its proteolytical removal leads to enzyme inactivation [9]. Evidence obtained from crystal structures of E. chrytsanthemi, E. corotovora, and Wolinella succinogenes L-asparaginases indicates that the flexible loop may be involved in substrate selectivity. The binding mode of different known ligands of L-asparaginases, such as L-Asp, L-Glu, D-Asp, and succinic acid monoamide is well conserved, with the binding of L-Glu accompanied by disorder of the flexible loop $(17,18,19)$.

Several asparaginase preparations are currently available for clinical use, including E. coli L-asparaginase II (EcA2), either native or conjugated with polyethylene glycol (PEG-Asparaginase), as well as an isoform extracted from Erwinia chrytsanthemi (ErA), available for patients with allergies to $E$. coli products. These products share the same therapeutic mechanism but differ considerably in their pharmacokinetics and immunological properties. EcA2 and ErA are both characterized by low in vivo stability due to the production of neutralizing antibodies and their protease susceptibility, leading to a rapid decline in Lasparaginase serum activity. Indeed, EcA2 and ErA have half-lives of just 30 and 
16 hours, respectively, meaning that several administrations are required for asparagine depletion [13]. Large variations in plasma activity have been associated with pronounced differences in the extent and persistence of L-Asn depletion, preventing significant leukemic cell death [14].

Despite the high remission rate achieved with the use of this enzyme, Lasparaginase based therapies are characterized by a high individual variation in pharmacokinetic and pharmacodynamic profile. More strikingly, independent reports have demonstrated that equivalent preparations of $\mathrm{L}$-asparaginase from different manufactures show substantial differences in enzyme half-lives, plasmatic activity and immunogenic profile despite having a similar sequence and in vitro activity $(8,9,10,11)$. Differences in the formulation of such a large and complex biotherapeutic drug can strongly impact the final biological product and compromise quality, safety, and efficacy with potentially radical clinical 110 consequences [19]. Since the macromolecular architecture plays a role in determining function and stability, a deep knowledge of the physicochemical landscape of similar biopharmaceuticals can provide anticipation of the product quality and clinical outcomes.

In this study, we investigated the oligomeric organization, stability, and dynamics of 115 EcA2 and its response to selective ligand binding. We used two brands of biosimilar EcA2 products, which further allowed the interpretation of the findings at light of the currently available information concerning their enzymatic activity in vivo and immunogenic profile $(10,11)$. 


\section{Material and Methods}

\subsection{Materials}

The EcA2 samples were kindly donated by the Martagão Gesteira Childcare and Pediatrics Institute (IPPMG-UFRJ), Rio de Janeiro, Brazil. We used lot \#F140328A 125 for Aginasa and lot \#2017010101 for Leuginase. The calculated molecular weight of mature asparaginase II from E. coli (Uniprot P00805; amino acids 23-348) was 34,593.94 $\mathrm{Da}$ for average mass and 34,572.56 $\mathrm{Da}$ for monoisotopic mass. The lyophilized powder containing EcA2 product was dissolved in water for injection (WFI) to $2,500 \mathrm{IU} / \mathrm{mL}$ and kept at $4{ }^{\circ} \mathrm{C}-8{ }^{\circ} \mathrm{C}$ prior to use in measurements. We used a conversion factor of about $255 \mathrm{IU} / \mathrm{mg}$ EcA2, based on the total potency declared in the product leaflet (https://www.accessdata.fda.gov/drugsatfda docs/label/2013/101063s5169lbl.pdf). No further corrections were performed. All other reagents were of analytical grade.

\subsection{Liquid chromatography - Electrospray ionization (ESI) - Mass spectrometry analysis of asparaginase products}

The asparaginase samples were diluted to $200 \mu \mathrm{g} / \mathrm{mL}$ in $0.1 \%$ formic acid in water. Each chromatographic run contained a total protein amount of $4 \mu \mathrm{g}(20 \mu \mathrm{L}$ protein solution per injection). Chromatographic analysis was performed in a Discovery BIO Wide Pore C18 column ( $15 \mathrm{~cm} \times 1 \mathrm{~mm}, 5 \mu \mathrm{m}$, Supelco, USA) in a Prominence

140 chromatographic system (Shimadzu, Japan) at $60^{\circ} \mathrm{C}$ and $100 \mu \mathrm{L} / \mathrm{min}$ mobile phase flux.

The elution was performed with a binary gradient $(A-0.1 \%$ formic acid in water; $B$ $-0.1 \%$ formic acid in acetonitrile) as follows:

- 0-1.5 min, $10 \%$ B

- $1.5-38.5 \min , 10 \%-60 \% \mathrm{~B}$

- Wash for 5 min with $95 \%$ B

- Re-equilibration with $10 \%$ B for 5 min

The detection was performed with a Diode Array (SPD-M20A Prominence, Shimadzu Co., Japan) scanning from 250 to $400 \mathrm{~nm}$, followed in line by a mass 150 spectrometer Impact (Bruker Daltonics, Germany) equipped with an ESI source, under the following conditions: gas nebulizer at 1.2 bar, drying gas flow at 8.0 $\mathrm{L} / \mathrm{min}$, capillary at $4.5 \mathrm{kV}$, source at $200^{\circ} \mathrm{C}$. The spectra were acquired in positive mode in the range of $\mathrm{m} / \mathrm{z} 250$ to 3,500 . The chromatogram and spectra were analyzed with DataAnalysis 4.2 (Bruker Daltonics, Germany) and mMass softwares 155 [20]. All analyses were performed at the Center for Biomolecular Mass Spectrometry (CEMBIO/UFRJ), Rio de Janeiro, Brazil. 


\subsection{Limited Proteolysis Assay}

L-asparaginase samples were diluted to a final concentration of $280 \mu \mathrm{M}$ and incubated at $25^{\circ} \mathrm{C}$ with $0.9 \mu \mathrm{g} / \mathrm{ml}$ of Proteinase K (Calbiochem, Cat\# 539480) in the 160 absence or presence of a 10-fold excess of L-Asp or L-Glu (2.8 mM). A sample was collected after different times of incubation, and the reaction was ended by heating at $100^{\circ} \mathrm{C}$ for 15 minutes in Laemmli loading buffer. The digestion profile was visualized by $15 \%$ SDS-PAGE stained with colloidal Coomassie blue. After 60 minutes, the reactions were frozen in liquid nitrogen and stored at $-80^{\circ} \mathrm{C}$ for further 165 analysis using liquid chromatography, Electrospray ionization, and mass spectrometry at the Center for Biomolecular Mass Spectrometry (CEMBIO/UFRJ), Rio de Janeiro, Brazil. LC-MS samples were thawed and diluted to a final concentration of $1.5 \mu \mathrm{M}$ in $0.1 \%$ formic acid in water and immediately injected in the LC-ESI-MS system. Each sample was submitted to two LC runs with different 170 detection methods, one performed without fragmentation in the range of 250-3,500 $\mathrm{m} / \mathrm{z}$ to obtain the molecular mass of the final products after limited proteolysis. For fragmentation experiments, spectra were recorded in the range of $50-1500 \mathrm{~m} / \mathrm{z}$ using the data-dependent acquisition mode (DDA/Auto-MS) with a three-precursor ion per fragmentation cycle.

175 2.4. Protein Enzymatic Digestion, MALDI-TOF-MS/MS Analysis, and Peptide de novo Sequencing.

After the intact protein mass analysis by both MALDI-TOF/MS and ESI Q-TOF/MS systems, we performed enzymatic digestion for both proteins with trypsin. Briefly, $100 \mu \mathrm{g}$ of Leuginase and Aginasa were independently incubated with a solution containing $20 \mathrm{mmol} / \mathrm{L}$ triethylammonium bicarbonate (TEAB), $0.1 \mathrm{mmol} / \mathrm{L}$ Tris $(2$ carboxyethyl) phosphine (TCEP), $40 \mathrm{mmol} / \mathrm{L}$ chloroacetamide at $45^{\circ} \mathrm{C}$ for 2 hours. The proteins were digested overnight with trypsin (1:50) (Promega, USA) in 1\% $(\mathrm{w} / \mathrm{v})$. The tryptic peptides were cleaned by using ZipTip TM C18 resin and submitted to MALDI-TOF/MS analysis using a saturated mixture of a-cyano-4-

185 hydroxycinnamic acid (CHCA), spotted on a MALDI sample plate, and dried at room temperature. Peptide fragmentation was obtained by MALDI-TOF-MS/MS experiments and the resulting data were analyzed manually using mMass software (37).

\subsection{Size Exclusion Chromatography}

190 SEC analysis was performed using a TSKgel G3000SW $\mathrm{XL}_{\mathrm{L}}$ column $(300 \times 7.8 \mathrm{~mm}$ Supelco) to determine the EcA2 hydrodynamic radius. We used samples of Aginasa, Leuginase, and Leuginase supplemented with L-Asp at 320, 150, 50, and $10 \mu \mathrm{M}$ in $10 \mathrm{mM}$ sodium phosphate at $\mathrm{pH}$ 7.0. Proteins were separated at a flow rate of $1 \mathrm{~mL} / \mathrm{min}$ and monitored by $\mathrm{OD}_{280 \mathrm{~nm}}$. Fractions of each peak were collected 195 and analyzed using 15\% SDS-PAGE stained with colloidal Coomassie blue to confirm EcA2 elution. The following globular proteins were used to fit a standard curve of elution profile versus hydrodynamic radius: thyroglobulin $86 \AA$ (SigmaAldrich, Cat \#T9145) bovine serum albumin-dimer $45.6 \AA$ and monomer $36.2 \AA$ 
(Sigma-Aldrich, Cat \#A7906), Ovalbumin $30.5 \AA$ (Sigma-Aldrich, Cat \#A5503), and carbonic anhydrase $21.4 \AA$ (Sigma-Aldrich, Cat \#C7025).

\subsection{Direct infusion ESI-traveling wave - ion mobility spectrometry - mass spectrometry (ESI-TWIM-MS)}

The asparaginase stocks were diluted to varying concentrations in different solutions (either $0.1 \%$ formic acid or a buffer comprised of $50 \mathrm{mM}$ ammonium acetate at $\mathrm{pH} 7.0,10 \%$ acetonitrile in the absence or the presence of L-amino acids in acid form), as indicated in the respective figure caption. Due to the lower signal, measurements performed in ammonium acetate were accumulated for up to $18 \mathrm{~min}$ at a flow rate of $20 \mu \mathrm{L} / \mathrm{min}$.

ESI-TWIM-MS measurements were performed in a Traveling Wave Ion Mobility 210 Mass Spectrometer (TWIM-MS, Synapt G1 HDMS, Waters, UK). The samples were directly infused at $20 \mu \mathrm{L} / \mathrm{min}$, using a positive ESI with a capillary voltage of $4.0 \mathrm{kV}$ and $\mathrm{N}_{2} \mathrm{~g}$ used as mobility gas at 0.4 bar. Data acquisition was conducted over the range of $\mathrm{m} / \mathrm{z} 500-3,000$ for 5 minutes. The mass spectrometer calibration was performed with $0.1 \%$ phosphoric acid (v/v) in acetonitrile: $\mathrm{H}_{2} \mathrm{O}(1: 1)$. Other

215 typical instrumental settings followed a previous study [21]. Data were analyzed according to [22] using DriftScope (Waters Corporation, UK). All measurements were performed at the Laboratory of Macromolecules (LAMAC) and Life Science Metrology Division (DIMAV) of of Brazil's National Institute of Metrology Standardization and Industrial Quality (INMETRO), Rio de Janeiro, Brazil.

\section{2.7. Protein Crystallography}

Crystals were obtained by sitting-drop vapor diffusion using $2 \mu \mathrm{L}$ protein solution with $2 \mu \mathrm{L}$ precipitant solution equilibrated against $80 \mu \mathrm{L}$ precipitant solution per well in a Corning 3225 multiwell plate. We used sparse matrix screening kits (Hampton Research, USA) and the recently prepared EcA2 formulations. The co225 crystallization of Leuginase with L-Asp was performed with a protein sample supplemented with $800 \mu \mathrm{M}$ L-Asp (final concentration) from a $20 \mathrm{mM}$ stock solution of amino acid in water. The crystals were obtained directly from the kit and used for data collection. Small needles/rods crystals were observed from assays with Leuginase within 5 min of setting up the crystallization screen, both in the presence 230 and absence of supplemented L-Asp.

The preliminary characterization of the crystals was performed at room temperature with crystals imbibed in Apiezon $\mathrm{N}$ and the $\mathrm{x}$-ray diffraction pattern using CuKa radiation ( $\mu \mathrm{S}$ 2.0, Incoatec) collected in a Photon II detector mounted in a D8-Venture diffractometer (Bruker AXS, Germany). Measurements were

235 carried out at the Laboratory for X-ray Diffraction - LDRX, Physics Institute, Federal Fluminense University (UFF), Rio de Janeiro, Brazil.

Several unsuccessful attempts were made to obtain crystals for both EcA2 formulations in the same crystallization condition. After characterizing the components of the formulations, we became aware that Leuginase contained 
240 mannitol and polyethylene glycol (PEG), which might have influenced the results from different crystallization conditions. The best diffraction quality crystals were obtained in:

Leuginase with or without L-Asp: $200 \mathrm{mM}$ magnesium acetate, 20\% PEG 3350 (Hampton Research PEG Ion I solution 25, "PEG ion I25")

245 Aginasa: 200 mM ammonium acetate, 20 \% PEG 3350 (Hampton Research PEG Ion I solution 30, "PEG ion I30")

Crystals were harvested from drops using $10 \mu \mathrm{m}$ nylon CryoLoop ${ }^{\mathrm{TM}}$ (Hampton Research, USA) and flash-frozen under an $\mathrm{N}_{2} \mathrm{~g}$ stream at $100 \mathrm{~K}$ and a flow rate of 10 L/h (Cryostream 700, Oxford Cryogenics, UK). Data collection was performed in 250 the MX2 beamline from the Brazilian Synchrotron Light Laboratory (LNLS) at Brazil's National Center for Research in Energy and Materials (CNPEM), Campinas, Brazil, using $1.459 \AA$ (8.4991 keV) radiation. Diffraction datasets were collected on Pillatus 6M detector (Dectris) with the following parameters:

Aginasa: PEG ion Screen I \#30, collection with exposure of 1 second/0.1 255 degree/image, 300 degrees (3,000 images) in total; structure solved by molecular replacement using 1IHD.pdb as a search model.

Leuginase supplemented with L-Asp: PEG Ion Screen I \#25, full X-ray diffraction dataset collection with an exposure of 3 second/0.1 degree/image, 270 degrees (2,700 images) in total; structure solved by molecular replacement using 1IHD.pdb 260 as a search model.

Leuginase apo: PEG Ion Screen I \#25, full X-ray diffraction dataset collection with an exposure of 0.5 second/0.1 degree/image, 180 degrees (1800 images) in total; structure solved by molecular replacement using 1IHD.pdb as a search model.

The images were indexed, processed, and integrated with XDS [23], and the molecular replacement was performed with 1IHD.pdb using the Python-based MX2-autoProc pipeline (developed by Dr. Andrey F. Z. Nascimento, CNPEM). The solved structures were further refined and rebuilt with C.O.O.T. [24], Refmac [25], and Phenix [26] using the mature EcA2 sequence (UNIPROT P00805). Water molecules were added using C.O.O.T. Structural validation of the models performed with CCP4 Suite v.6.0.2 showed that almost all dihedral angles are within the favored range. Figures were generated with PyMOL (PyMOL Molecular Graphics System, Version 2.0, Schrödinger LLC). Selected crystallographic parameters are found in Table S3. The resulting models were deposited under pdb codes 6UOH (Aginasa, co-crystallized with L-Asp), 6UOD (Leuginase - apo), and 275 6UOG (Leuginase co-crystallized with L-Asp). Data collection and refinement statistics are found in Table S3.

The estimative of the intermolecular distances between EcA2 catalytic site residues and the ligand L-Asp as well as Val27 intramolecular distances was performed with MolMol V1.0.7 (Koradi et al. 1996) using a maximal distance of $5 \AA$. The list of distances obtained with this analysis is summarized in Table S5 and S6. 


\subsection{NMR spectroscopy}

Nuclear Magnetic Resonance (NMR) measurements were performed at $25^{\circ} \mathrm{C}$ on a $400 \mathrm{MHz}$ Bruker Avance III spectrometer equipped with a broadband inverse Zaxis gradient probe (Bruker Spin Corp., $\mathrm{GmbH}$ ). ${ }^{1} \mathrm{H}$ chemical shifts were

285 referenced to 3-(trimethyl-silyl)-1-propanesulfonic acid, sodium salt (DSS). The ${ }^{13} \mathrm{C}$ chemical shifts were indirectly referenced to DSS by using the absolute frequency ratios. All NMR spectra were processed and analyzed with Topspin 4.0.6 (Bruker Spin Corp., GmbH).

The NMR samples used to characterize the EcA2 formulation products were

290 ultrafiltrated using Amicon (Millipore) with a $3 \mathrm{kDa}$ cut-off to remove the protein from the solution. The flow-through, supplemented with $10 \%$ deuterium oxide, was used to acquire 1D ${ }^{1} \mathrm{H}$ spectra with water suppression using excitation sculpting with gradients. The $2 \mathrm{D}\left[{ }^{1} \mathrm{H},{ }^{13} \mathrm{C}\right]-\mathrm{HSQC}$ spectra were collected using the whole formulation with $10 \%$ deuterium oxide. The spectra obtained were compared with 295 the ones acquired for standard solutions of mannitol, L-Asp, and PEG. For the quantitative analysis, we acquired several $1 \mathrm{D}{ }^{1} \mathrm{H}$ spectra with serial dilutions ranging from 1,000 to $62.5 \mu \mathrm{M}$ of L-Asp and 20 to $1.25 \mathrm{mg} / \mathrm{mL}$ of mannitol. The peak areas were integrated and used to generate a standard curve with the concentration of each compound.

300 NMR samples used for the amino acid titration analysis were prepared with $160 \mu \mathrm{M}$ of EcA2 (Leuginase) in $10 \mathrm{mM}$ sodium phosphate at $\mathrm{pH} 7.0$, with the addition of the respective amino acid, for the acquisition of $1 \mathrm{D}{ }^{1} \mathrm{H}$ NMR spectra. The intensities observed for $\mathrm{Y} 25 \mathrm{H}^{\varepsilon}$ and $\mathrm{H}^{\delta}$ signals, at 6.7 and $7.0 \mathrm{ppm}$, were normalized against a resonance at approximately $6.8 \mathrm{ppm}$ since no intensity variation was found for this 305 signal in the presence of the substrates.

The STD-NMR experiments were performed at $298 \mathrm{~K}$ on a Bruker Avance III spectrometer operating at ${ }^{1} \mathrm{H}$ frequencies of $600 \mathrm{MHz}$ equipped with a tripleresonance, Z-axis gradient $5 \mathrm{~mm}$ probe. The EcA2 used for these experiments were previously desalted in a 5-mL HiTrap Desalting (GE Healthcare, USA) and

310 eluted in $10 \mathrm{mM}$ sodium phosphate at $\mathrm{pH}$ 7.0. The samples were prepared with a 500 -fold excess of ligand (L-Asp or L-Glu $20 \mathrm{mM}$ ) over protein, $40 \mu \mathrm{M}$. The onresonance spectrum was obtained with the saturation of protein signals at 0.87 ppm and the off-resonance spectrum at $10 \mathrm{ppm}$, using the Bruker standard parameters (STDDIFFGP.3). A spin-lock filter of $25 \mathrm{~ms}$ was used to suppress

315 protein signals. A saturation time build-up curve was obtained to evaluate if an accumulation of saturated ligand molecules was occurring. The saturation times used were $0.5,1.0,1.5,2.0,2.5,3.0,3.5$, and $4.0 \mathrm{~s}$ with recycling delay of $4.0 \mathrm{~s}$. STD amplification factors ( $A_{\text {STD }}$ ) were determined using the following equation [27]:

$$
A_{S T D}=I_{S T D} / I_{0} \times[L] T /[P]
$$

320 where $[\mathrm{L}]_{T} /[\mathrm{P}]$ is the molar ratio of the ligand in excess relative to the protein, and $\mathrm{ISTD}_{\mathrm{ST}} / \mathrm{l}_{0}$ is the ratio of the ligand signal integrals when the saturation is on-resonance $\left(\mathrm{I}_{\mathrm{SDT}}\right)$ or off-resonance $\left(\mathrm{I}_{0}\right)$. The relative STD percentages were obtained using 2.5 
s for saturation. The highest intensity of $A_{\text {STD }}$ for each ligand was set to $100 \%$, and all other STD signals were calculated relative to this.

\subsection{Circular Dichroism}

Circular dichroism experiments were performed on a Chirascan ${ }^{\mathrm{TM}} \mathrm{CD}$ spectropolarimeter (Chirascan, Applied Photophysics, Leatherhead, UK) with a 100 $\mu \mathrm{m}$ path-length quartz cuvette. Far-UV spectra were recorded from 190 to $260 \mathrm{~nm}$ using $\mathrm{EcA} 2$ at $15 \mu \mathrm{M}$ diluted in $10 \mathrm{mM}$ sodium phosphate at $\mathrm{pH} 7.0$ in the absence or presence of $1 \mathrm{mM} \mathrm{L-Asp}$ with a spectral resolution of $0.5 \mathrm{~nm}$, a scan speed of 10 $\mathrm{nm} / \mathrm{min}$, and an average of three accumulations. The final data were obtained after subtraction of the buffer signal for background correction and are reported as molar residue ellipticity (deg. $\mathrm{cm}^{2} \cdot \mathrm{dmol}^{-1}$ ), calculated as described in [28]. The thermal denaturation curve was monitored at $220 \mathrm{~nm}$ while the sample was heated from $25^{\circ} \mathrm{C}$ to $80^{\circ} \mathrm{C}$ in increments of $0.5^{\circ} \mathrm{C}$ using a rate of temperature change of $1^{\circ} \mathrm{C} / \mathrm{min}$. The data were processed using Prism 6 (GraphPad Software, USA). The melting temperature $\left(T_{m}\right)$, determined as the inflection point and slope of the sigmoidal curve, was calculated using a Boltzmann sigmoid equation.

\subsection{Small-angle X-ray scattering (SAXS)}

340 SAXS measurements were performed at $25^{\circ} \mathrm{C}$ in the D01A/SAS1 beamline from the LNLS/CNPEM equipped with a Pilatus $300 \mathrm{~K}$ detector following [29]. Samples were directly measured in a mica sample holder (1 $\mathrm{mm}$ optical path) and adequately subtracted for blank after total attenuation and photon counting. The samples were exposed for $300 \mathrm{sec}$ at $1.488 \AA$, and no radiation damage was

345 inferred when compared to shorter exposures. The scattering pattern was integrated with Fit2D [30], and ATSAS Suite 2.8.2 [31] was used in data analysis. The oligomeric distribution was calculated with Oligomer [32] using form factors calculated with FFMaker and the structural models designed from the EcA2 from Elspar (PDB ID 3ECA) using PyMOL in the q range of $0.022 \mathrm{~A}^{-1}$ to $0.48 \mathrm{~A}^{-1}$. 


\section{Results}

\subsection{The dissimilar formulations of EcA2 products}

The analysis of the intact EcA2 products by LC-MS revealed a major peak in the chromatograms of Aginasa (Fig. 1A) and Leuginase (Fig. 1B), whose charge envelopes (Fig. 1C and 1D) allowed the estimation of their molecular mass at $34,590 \mathrm{Da}$ and $34,576 \mathrm{Da}$, respectively (Fig. 1E). We have no objective explanation for the $15 \mathrm{Da}$ difference between them. The leaflets of both products do not indicate any particular feature, such as post-translational or chemical modifications, which would be the most obvious explanations for such a difference.

360 In order to confirm the sequence identity of the protein we further performed enzymatic digestion of Aginasa and Leuginase with trypsin, followed by peptide identification by MALDI-TOF/TOF mass spectrometry. We found fragments corresponding to the predicted sequence of asparaginase for both Aginasa and Leuginase proteins (Fig. 1B), and the MS/MS spectra assignment for Aginasa and

365 Leuginase $[\mathrm{M}+\mathrm{H}]+2028.09 \mathrm{~m} / \mathrm{z}$ allowed the corrected sequencing of the $\mathrm{N}$-terminal 22 residues fragment (Fig. S1C).

We found a smaller peak in the Leuginase chromatogram (Fig. 1B), which, according to ESI-MS, corresponds to polyethylene glycol with an average mass of $6 \mathrm{kDa}$ (Fig. S2). We performed a 2D $\left[{ }^{1} \mathrm{H},{ }^{13} \mathrm{C}\right]-\mathrm{NMR}$ analysis and confirmed the

370 presence of a PEG in Leuginase (Fig. $2 \mathrm{~B}$ ), as indicated by a major peak in its spectrum at about $3.6 \mathrm{ppm}\left({ }^{1} \mathrm{H}\right)$ and $69.5 \mathrm{ppm}\left({ }^{13} \mathrm{C}\right)$, which matches a test spectrum of PEG alone (Fig. 2D). In the NMR measurements, we identified other peaks at low field, which clearly correlate to mannitol (Fig. 2C). No peaks corresponding to PEG or mannitol were detected in Aginasa. However, other NMR

375 peaks found in Aginasa but not in Leuginase correspond to free aspartate, at about $260 \mu \mathrm{M}$ (ASP, which NMR could not distinguish between L and D forms) (Fig. 2A). The asparaginase product Elspar ${ }^{\mathrm{TM}}$,, which was used to obtain the first crystal structure of E. coli Asparaginase II (PDB ID 3ECA) [7], was reported to contain ASP, while its leaflet disclosed that its formulation included mannitol 380 (https://www.accessdata.fda.gov/drugsatfda docs/label/2002/aspamer080102LB.p df). In opposite, Leuginase contains PEG and approximately $10 \mathrm{mM}$ mannitol but no detectable ASP, as estimated from analytical curves (concentrations derived from quantitative NMR analysis shown in Fig. S3).

\subsection{The thermodynamic stability of EcA2}

To gain access to the overall conformation and stability of EcA2, we performed a thermal denaturation following changes in secondary structure by circular dichroism. We observed a large pre-transition plateau for both EcA2 products, with an abrupt transition accompanying a substantial change in the signal at $220 \mathrm{~nm}$ 390 (Fig. 3). Both formulations presented similar Tm $\left(67.52 \pm 0.10^{\circ} \mathrm{C}\right.$ for Aginasa and $67.87 \pm 0.13^{\circ} \mathrm{C}$ for Leuginase). No apparent changes in their melting curves were 
observed when the experiment was performed in the presence of L-Asp (Tm of $66.78 \pm 0.08^{\circ} \mathrm{C}$ for Aginasa plus L-Asp and $67.91 \pm 0.06^{\circ} \mathrm{C}$ for Leuginase plus LAsp). These thermodynamic data suggest a minor contribution of ligand binding

395 and conformational change over broader folding energetics of the EcA2 against thermal denaturation. The most evident difference between the formulations was the slope for their thermal transition. Leuginase had slopes of $0.39 \pm 0.02$ and 0.44 \pm 0.02 , with and without the presence of $260 \mu \mathrm{M}$ of L-Asp, respectively. These slopes were subtler for Aginasa (0.21 \pm 0.02 and $0.22 \pm 0.02$, respectively).

400 3.3. The oligomeric distribution of asparaginase by small-angle $X$-ray scattering (SAXS) and size-exclusion chromatography (SEC)

We performed SAXS measurements of the two EcA2 products to gain insight into the oligomeric distribution of EcA2. The scattering profile for each EcA2 product was similar between different vials reconstituted independently for Aginasa (Fig.

405 4A) and Leuginase (Fig. 4B). Attempts to deconvolute the oligomeric fraction from EcA2 Aginasa were not successful, even when oligomeric models of octamers or higher-order oligomers were included (Fig. S5). However, the deconvoluted scattering curves of Leuginase, revealed $81 \%$ tetramers, $10 \%$ trimers, $9 \%$ dimers, and no detectable monomers (Fig. 4E). Dilution of Leuginase up to 10 times 410 showed no major effect on the presence of tetramers as a principal component in the oligomeric distribution of Leuginase (Fig. 4D).

To further characterize the oligomeric distribution of EcA2, we performed SEC using TSKgel G3000SW $\mathrm{XL}$ (Fig. 5). We observed main peaks for Aginasa and Leuginase that were surrounded by minor peaks with distinct hydrodynamic radii,

415 according to the standards used (Fig. S6 and Table S2). All marked peaks correspond to EcA2 for both products, as revealed by SDS-PAGE of the fraction collected from the chromatographed samples, suggesting that differences in the chromatographic peaks are likely to be related to oligomeric distribution or conformational fluctuations. The SEC profiles of both products are mostly not

420 altered by dilution from $320 \mu \mathrm{M}$ down to $10 \mu \mathrm{M}$ or by the addition of L-Asp, confirming the SAXS findings for diluted samples (Fig. 4 and Fig. S7). The most noticeable difference is the relative abundance of fraction F3 and a deviation of the chromatogram of Aginasa. Given that the EcA2 substance is the major constituent of both products as indicated by LC-MS (Fig. 1), we hypothesize that the peak

425 observed in Aginasa, with a retention time of $7.1 \mathrm{~min}$, might be an indicator of higher-order assemblies. It is worth noting that the main peak of Leuginase presented a higher apparent elution time $(8.7 \mathrm{~min})$ in comparison to Aginasa (8.3 $\min )$.

\subsection{The polymorphic distribution of Asparaginase and ligand interaction by ESI-TWIM-MS}

We further explored the polymorphic distribution of EcA2 in solution using ESITWIM-MS. This method separates gas-phase ions based on their size, 3D shape, 
and ion-neutral interactions while the ions travel through a drift gas under the influence of an electric field. Studies have shown that this method is robust in determining the oligomeric and conformational distribution of proteins $(20,21)$.

The deconvolution of the integrated spectra showed a mass difference of about 15 Da between the EcA2 products (Fig. 6), which independently confirms the results from intact LC-ESI-MS (Fig. 1). Both EcA2 products, Aginasa (Fig. 6A) and 440 Leuginase (Fig. 6B), presented two populations in the ESI-TWIM-MS. The analysis of the stripped spectra corresponding to each of the two populations revealed their equivalence in molecular mass (Fig.s 6C and 6D). These data suggest the existence of EcA2 monomers in two defined populations of conformers.

ESI-TWIM-MS analysis of EcA2 in the presence of L-Asp or L-Glu revealed evidence for the amino acid-bound monomer (Fig. 7B and 7D) in varying charged states $(M+15$ to $M+12)$ with a single distribution along the mobility axis (Fig. S8), indicating the distribution along with a well-defined population. Measurements performed in the presence of Gly showed a similar profile to the apo form (Fig. 7C), indicating the lack of detectable complex with this amino acid and ruling out nonspecific EcA2: L-aa interaction by adduct effect. Collectively, these data demonstrate the selective nature of ligand interaction with EcA2 monomers.

\subsection{The structural basis for amino acid interaction with the EcA2 in the crystal phase}

To gain more detailed insights on the structural basis of EcA2 interaction with ASP,

455 we pursued the elucidation of the crystal structure of both EcA2. We solved the structures by obtaining diffracting quality crystals. The monomeric units within their asymmetric units were similar between the three structures, with overall RMSD of $1.2 \AA$ or lower (Table S4). An electron density blob was observed within the catalytic site of Aginasa, which we attributed to L-Asp since NMR indicated the 460 presence of this amino acid in the formulation (Fig. 2). In contrast, Leuginase was found in an 'open' conformation, without ligands in the active site and with only part of the N-terminal active site lid sufficiently visible for modeling, while Aginasa showed a well-ordered lid (Fig. 8). Attempts to crystallize Leuginase supplemented with L-Asp, resulted in cocrystallization of EcA2 with L-Asp in the active site and

465 showing a closed lid, in close identity to the Asp-bound EcA2 from Aginasa (Fig. 8 and Table S4). These data demonstrate that EcA2 is structurally responsive to the interaction with L-Asp and the resulting consolidated crystal structural model of the two formulations are equivalent.

\subsection{The dynamics of the active site lid using NMR}

NMR revealed further insights on the dynamics of the active site lid upon binding to amino acids. 1D ${ }^{1} \mathrm{H}-\mathrm{NMR}$ spectra of the apo form of EcA2 (Leuginase) revealed two sharp peaks at $6.7 \mathrm{ppm}$ and $7.0 \mathrm{ppm}$ and three broad peaks at 7.7-8.4 ppm, not observed in the holo form of EcA2 (Aginasa) (Fig. 9A). The two sharp peaks 
475 were previously attributed to $\mathrm{H}^{\varepsilon}$ and $\mathrm{H}^{\delta}$ of the aromatic ring of Tyr25, which belongs to the loop region comprising the lid over the active site of EcA2, and has been shown to be detectable in the apo form due to high conformational flexibility [35]. According to this interpretation, in case this loop gets more rigid, the peaks would get broader and fade out within the other broad signals in the spectra. Indeed, as observed in the EcA2 structure with bonded L-Asp, these signals disappear indicating the $\mathrm{N}$-terminal loop closure in the presence of L-Asp or L-Asn. These changes were not observed when L-Glu or L-Gln were added to EcA2, indicating the selectivity of EcA2 for L-Asn and L-Asp for the induction of conformational changes in the loop region comprising the Tyr25, ultimately resulting in a closed form of the active site. The spectra do not present clear indication of alteration in the equilibrium of oligomerization of EcA2 in any of these samples, which would be represented by a global change in the line widths (Fig. S9). Instead, for example, there are peaks at 6.2, 6.6, 7.2 and $7.6 \mathrm{ppm}$, which mostly did not change their width.

490 To further investigate the selectivity of EcA2 for L-Asn/L-Asp over L-GIn/L-Glu and their ability to induce a conformational response, we performed titration analyses monitoring the aromatic resonances attributed to Tyr25 of EcA2 by 1D ${ }^{1} \mathrm{H}-\mathrm{NMR}$. The titration with L-Asp (Fig. 9B) indicated that this ligand restricted the loop flexibility in an EcA2:L-Asp ratio of about 1:1, evidenced by the disappearance of a pair of sharp Tyr25 signals. When EcA2 was titrated with L-Glu (Fig. 9C), a significant change in Tyr25 resonances could only be detected with an EcA2:L-Glu ratio of over 1:300. Even with a 1:1800 proportion of ligand, the observable intensities of Tyr25 resonances were approximately $50 \%$ of the initial intensity prior to the addition of the ligand (Fig. 9D). This demonstrates the inability of L-Glu to

500 induce conformational changes in EcA2 to the same extent as L-Asp.

\subsection{L-Asp protects EcA2 against limited proteolysis of the active-site lid}

To further evaluate the selective effect of amino acid-binding on the active site lid in the presence of ligand binding in the catalytic site, we tested the influence of LAsp or L-Glu over the propensity of EcA2 for limited proteolysis. The resulting 505 products from the proteolysis were subjected to identification by LC-ESI-MS, which revealed two major chromatographic peaks as proteolytic products, one corresponding to an ion equivalent in mass to the $\mathrm{N}$-terminal residues 1-23 of EcA2 and the other to the remaining part of the protein (Fig. S8).

The EcA2 was incubated with proteinase-K in the absence and presence of L-Asp

510 or L-Glu, and aliquots were taken at varying time intervals and subjected to SDSPAGE. The EcA2 product Leuginase showed a time-dependent decrease in the main protein band corresponding to the unaltered protein with the progressive increase of a band with higher electrophoretic mobility (Fig. 10). The EcA2 product from Aginasa, which was found to contain an equimolar amount of ASP, showed

515 slower proteolysis kinetics. Supplementation of both both Aginasa and Leuginase with further amount of L-Asp resulted in a decreased rate of degradation when compared to the assay conducted in the original EcA2 preparations. No protection 
was detected when the limited proteolysis was conducted in the presence of L-Glu. These data independently confirm the selective nature of ligand binding to the active site and its influence on the dynamics of the active site lid.

\subsection{Contact points and affinity of amino acid interaction with EcA2 in solution by STD-NMR}

In addition to our evaluation of the selective nature of ligand binding on the

525 dynamics of the active site lid of EcA2, we used STD-NMR to characterize these effects on the ligand. This method allows the recognition of the binding epitope on the ligand through the efficiency of saturation transfer from the protein that is saturated specifically by a long radio-frequency pulse. The efficiency of the saturation transfer is quantitatively expressed by the amplification factor (A $A_{\text {STD }}$ ).

530 The intensity of $A_{S T D}$ characterizes the average number of transient contacts $(\leq 5$ $\AA$ ) of the ligand per molecule of receptor in a given saturation time [27].

The plot of the ASTD at different saturation times obtained for L-Asp demonstrates that $H^{\alpha}$ exhibits a remarkably stronger $A_{S T D}$ than $H^{\beta 2}$ and $H^{\beta 3}$ (Fig. 11A). The relative STD percentages indicate that the $\mathrm{H}^{\alpha}$ is closer to EcA2, while the $\mathrm{H}^{\beta}$ is more distant.

Additionally, we conducted STD-NMR experiments for L-Glu as an attempt to better describe the mechanism of EcA2 ligand recognition (Fig. 11B). Despite presenting a weaker STD signal due to a higher $K_{\mathrm{M}}$ for this ligand [36], the $A_{\text {STD }}$ plot obtained for L-Glu shows relative STD percentages of $100 \%, 70.2 \%$, $54048.9 \%$, and $54.2 \%$ for $\mathrm{H}^{\alpha}, \mathrm{H}^{\beta}, \mathrm{H}^{\gamma^{2}}$, and $\mathrm{H}^{\mathrm{\gamma}}$, respectively, indicating a weaker and more isotropic interaction of L-Glu in comparison to L-Asp. 


\section{Discussion}

\subsection{Structural and Dynamic Aspects}

Asparaginase has long been described as a specific hydrolase for L-Asn, although the enzyme activity is also reported for L-Gln. In fact, our data reveal the propensity of EcA2 to bind to L-Asp and L-Glu, though not to Gly (Fig. 7, ESITWIM-MS). L-Asp induces conformational changes to close the active site lid (Fig. 8 and 9). Furthermore, L-Asp, but not L-Glu, is selective in changing the active site dynamics, as shown in our study by Tyr25 modulation (Fig. 9), limited proteolysis (Fig. 10), and binding measured by STD-NMR (Fig. 11). Specific ligand binding is thus a process that involves the final consolidated state, overall affinity, and kinetic processes.

555 EcA2 has been described as a tetramer $(17,28)$. While a tetramer can be observed by SAXS (Fig. 4), as well as by crystallographic organization, our ESI-TWIM-MS data show the selective interaction of L-Asp and L-Glu with monomeric EcA2. Interestingly, the abundance of a tetramer is not apparent in the SEC-HPLC analysis (Fig. 5), where the main species has a calculated hydrodynamic radius of 560 31.2-34.8 A (Table S2), which is most likely indicative of a dimeric state of EcA2. The interaction of EcA2 with ligands as monomers could be a result of enzymatic activity in vivo observed for hours after injection; this causes the extensive dilution of the protein and could favor its dissociation. However, the $K_{d}$ of the tetramer and dimer in such biological milieus is unknown.

565 EcA2 has an N-terminal flexible loop that can form a species of lid for its active site (residues 10-37). This lid contains a cleavage site between S23 and N24 that is an important locus of EcA2 cleavage by lysosomal proteases, responsible for the in vivo inactivation of the enzyme [38]. N24 is located at the N-terminal flexible loop, which in Aginasa has reduced flexibility in the presence of L-Asp bound to the 570 active site, causing it to remain in its closed conformation. The increased protease accessibility of N24 and, consequently, the higher propensity for proteolysis demonstrated by Leuginase is most likely related to the open-loop conformation observed for the EcA2 present in this product. With the addition of L-Asp, Leuginase exhibits a degradation profile similar to Aginasa, indicating that L-Asp

575 binding exerts a protective effect against proteolysis. We did not detect any additional protection against proteolysis after incubation with L-Glu. These observations confirm the results obtained by NMR, demonstrating the inefficiency of L-Glu in stabilizing the EcA2 closed-lid conformation.

The measurement of the distances between L-Asp and the residues that constitute 580 the catalytic site of EcA2 (Table S5), along with the STD-NMR data, revealed that $\mathrm{L}$-Asp $\mathrm{H}^{\alpha}$ is in close contact with $\mathrm{H}^{\mathrm{\gamma} 21}$ of $\mathrm{V} 27$, located at the $\mathrm{N}$-terminal loop of EcA2 (Fig. 11 and 12). L-Glu causes no more than $50 \%$ of the conformational effect in the loop, likely reflecting the much lower affinity of this amino acid for EcA2 (1.9 x 10 ${ }^{-5} \mathrm{M}$ for L-Asn vs. $5.9 \times 10^{-3} \mathrm{M}$ for L-GIn) [36]. In addition to its direct 
585 role in ligand binding stabilization, Val27 is also in close contact with Gly11, part of the $\mathrm{N}$-terminal hinge of the loop that allows this element to transit between conformations upon ligand binding [12], limiting hinge mobility. Contacts of Val27 with the loop residue Tyr25 were observed and possibly contributes with the restriction of Tyr25 sidechain flexibility, as observed by NMR. Moreover, as 590 identified in the crystal structure, the residue Val27 interacts with Gly57, which is part of the active site (Table S6).

Sequence alignment of different species of L-asparaginase using $\operatorname{BLASTp}(31,32)$ was highly conserved for the residues involved in binding and catalysis (Fig. 13). Interestingly, Val27 is present only in EcA2 and L-asparaginase from 595 Saccharomyces cerevisiae, while other L-asparaginases have alanine or serine at this position. A previous study using EcA2 variants with mutations on V27 replaced with the apolar amino acids Met and Leu showed a decrease in catalytic activity for L-Asn and L-GIn to about $20 \%$ of the wild type activity due to a reduced $\mathrm{K}_{\text {cat }}$ value.[41]. Another study carried out with engineered ErA2 variants with mutations

600 in A31, the corresponding residue to EcA2 V27 in this isoform, demonstrated substantial variations in substrate affinity and L-asparaginase/L-glutaminase activity [5]. The crystallographic structure of ErA2 A31I, a variant with higher affinity for both L-Asn and L-GIn, revealed that this mutation forced a different conformation in the $\mathrm{N}$-terminal flexible loop in comparison to the wild-type enzyme.

605 However, the authors could not explain the decreased $K_{M}$ value observed for this mutant since no obvious conclusion could be made from the structure and they speculated that this mutation influenced the dynamics of the loop clorsure and ultimately the substrate affinity.

The superposition of the L-asparaginase catalytic site structure shows that 610 stereochemical features are also highly conserved, and the main difference lies in the orientation of the loop forming the lid over the catalytic site (Fig. 14). This is an intrinsic characteristic that affects the catalytic activity and substrate specificity of these enzymes, with EcA2 having one with the lowest $K_{M}$ values of the enzymes studied $(34,35,36)$, despite the similarities in sequence and overall folding.

615 These observations indicate that Val27 plays a crucial role in the conformational changes in the lid following substrate binding and stabilization of the catalytic site conformation. This could explain the differences in catalytic activity, substrate affinity, and loop conformation exhibited for different L-asparaginases. This residue is then considered a good target for enzyme engineering.

620 4.2. Formulation aspects

We found that both Aginasa and Leuginase had molecular weights that are similar and compatible with the mature form of EcA2 (Uniprot P00805; amino acids 23348 ), with an average mass of $34.593,94 \mathrm{Da}$ and monoisotopic mass of $34.572,56$ Da. We performed MS analysis of both products under two entirely different setups

625 and spectrometers and elucidated their crystal structures from each dataset. All data showed redundant evidence for mature EcA2 as the major component of the formulation. Previous work reported molecular weight of $36.8 \mathrm{kDa}$ for Leuginase, 
which would correspond to the full amino acid sequence of EcA2, including the 22amino acid long-signal peptide [18]. However, data from tandem-mass 630 spectrometry sequencing from this same study did not find the complete signal peptide. We have no reasonable explanation for these conflicting results except for the fact that we used different batches. However, it seems implausible that the production method and the resulting biopharmaceutical product would have changed within such a short interval (the difference between batches was 635 approximately one year). Unfortunately, the manufacturing processes of synthetic and biosynthetic pharmaceutical products are proprietary information, which is only made available to regulatory agencies in documents such as the Drug Master Files (DMFs) or New Drug Applications (NDAs) of the U.S. Food and Drug Administration (FDA).

640 Interestingly, we found that the EcA2 in Leuginase has an average mass of $15 \mathrm{Da}$ less than expected. This difference between Leuginase and Aginasa suggests the presence of different amino acid sequences that were not detected within the coverage limits of the mass spectrometry proteolytic fragmentation analysis. Although some set of amino acid substitutions could result in a 15 Da difference, it 645 would be counterproductive to engage in conjecture. Given that both products show similar activity in vitro $(10,11,25)$ and similar physicochemical and ultrastructural properties as described in this study, we assume that this difference may represent a molecular signature to identify the compound, although we cannot confirm whether this is intentional or unintentional.

650 The product leaflets do not typically disclose the components of the formulation; non-guided analysis of the product often results in such components being found. For example, L-Asp has been found in the crystallographic structures of the native EcA2 formulation Elspar [7] and the Y25F variant of EcA2 [46]. Since neither compound was incorporated as soaking/co-crystallization, the L-Asp was part of

655 the product formulation. We found both mannitol and PEG in Leuginase, which could be present due to purification by crystallization [47] or to increase the stability of the formulation. The product leaflet, however, does not disclose any adjuvants in the formulation. Furthermore, we found aspartate in Aginasa, which is also not disclosed in its leaflet. All these compounds are justifiable from a downstream 660 perspective, including during final product stabilization. However, the lack of full disclosure of their composition and the implications for their in vitro activity/physicochemical behavior hamper the development of a pharmacopeic monograph, which is only currently available for EcA2 in the Chinese Pharmacopoeia (Office TS 2017, ), with no mention of monitoring for related 665 substances including PEG, mannitol, aspartate, sulfate or any other stabilizer or residual component of the downstream process. Moreover, both products Aginasa and Leuginase - entered the Brazilian health care system through an international purchase conducted by the Ministry of Health. In addition, no bidding process was required for the use of this drug in Brazil. Based on our findings, we 670 suggest that a drug master file must be made available to regulatory agencies, along with clinical data and full disclosure of the components in the pharmaceutical product leaflet. Doing so would provide greater awareness of the products being 
used and would ensure fuller transparency to the clinicians and health care system.

\subsection{Further therapeutic aspects}

EcA2 has long been used as a therapeutic agent with impressive results in the success rate of ALL treatment $(37,38)$. However, some patients are not responsive to this therapy, perhaps owing to the inactivation of the enzyme by immune response or the proteolytic degradation of EcA2 [51]. It is worth noting that this erratic response to therapy is also observed with the use of PEGylated EcA2 [38]. In this context, the development of other L-asparaginases with higher in vivo stability against inactivation and higher catalytic efficiency may benefit from the conformational and dynamic features unveiled in this work. However, this is not a complete mapping of the conformational and stability landscape of EcA2. Further studies are advised with an extended mapping of the interaction with other amino acids, as well as long-term stability studies in the presence of the amino acids selected as the best candidates for stabilization (most likely L-Asp).

Despite the similar conformational and stability profile of Aginasa and Leuginase in the presence of L-Asp as shown by IMS, crystal structure, NMR measurements, and partial proteolysis, we are still intrigued by the $15 \mathrm{Da}$ difference between them. This shall merit further detailed investigation since, as far as we are aware, no drug master file has been disclosed. It remains to be determined whether this difference is related to the subtle, but significant, differences in their thermostability and hydrodynamic radii.

695 Our results demonstrate that the selectivity of L-asparaginase is correlated with the stabilization of its active site lid conformation. In light of our results and the accumulated evidence from the literature, the selectivity of L-Asp in binding and its effect on the dynamics of the active site lid support its use as a kinetic and thermodynamic stabilizing agent of this enzyme.

700 In addition, the pharmacopeial monograph for EcA2 in the Chinese Pharmacopoeia (Office TS 2017), determine specifications assays such as identification by high performance liquid chromatography, $\mathrm{pH}$, purity assessed by size exclusion chromatography, and enzymatic activity to determine potency in vitro. However, these techniques provide information that corresponds to a global average of the

705 protein structure and chemical identity and subsequently are usually uncapable to detect changes in amino acid sequence and specific conformational changes. Therefore, a more detailed physical-chemical characterization and structural investigation, using analytical techniques with higher accuracy and resolution such as NMR and MS of the final product is imperative to avoid harsh clinical disparities. 
bioRxiv preprint doi: https://doi.org/10.1101/2020.11.11.379065; this version posted November 12, 2020. The copyright holder for this preprint (which was not certified by peer review) is the author/funder. All rights reserved. No reuse allowed without permission.

Data availability: The crystallographic structure factors and pdb models presented in this paper have been deposited in the Protein Data Bank (PDB) with the following codes: $6 \mathrm{UOD}, 6 \mathrm{UOG}, 6 \mathrm{UOH}$. All other datasets generated during and/or

715 analyzed during the current study are available from the corresponding author on reasonable request. 


\section{Acknowledgments}

We acknowledge Dr. Rafael Andrade and Jessica Moreira for their technical 720 support on protein biochemistry at Plataforma Avançada de Biomoléculas/UFRJ. We would like to thank LNLS-CNPEM (MX2 and SAS1 beamlines), UEMP-UFRJ, CENABIO-UFRJ, CEMBIO-UFRJ, LDRX-IF-UFF and LAMAC-DIMAV-INMETRO and their staff for the access to their analytical facilities. This research used resources of the Brazilian Synchrotron Light Laboratory (LNLS), an open national

725 facility operated by the Brazilian Centre for Research in Energy and Materials (CNPEM) for the Brazilian Ministry for Science, Technology, Innovations and Communications (MCTIC).

\section{Funding and additional information}

730 This study was supported by Fundação Carlos Chagas Filho de Amparo à Pesquisa do Estado do Rio de Janeiro (grants E-26/202.998/2017-BOLSA, to LMTRL and E-26/290.076/2017 to MSA), by the Conselho Nacional de Desenvolvimento Científico (grant PQ2/311582/2017-6, to LMTRL), by the Coordenação de Aperfeiçoamento de Pessoal de Nível Superior (CAPES, Finance Code \#001) and the Programa Nacional de Apoio ao Desenvolvimento da Metrologia, Qualidade e Tecnologia (PRONAMETRO, to LMTRL) from the Instituto Nacional de Metrologia, Qualidade e Tecnologia (INMETRO). The funding agencies had no role in the study design, data collection and analysis, or decision to publish or prepare of the manuscript.

Conflict of Interest: The authors have no financial conflicts of interest with the contents of this article. LMTRL is a participant in patent applications by the UFRJ on controlled release of peptides unrelated to the present work. The remaining authors have nothing to disclose. 


\section{References}

745 [1] S. Patil, J. Coutsouvelis, A. Spencer, Asparaginase in the management of adult acute lymphoblastic leukaemia: Is it used appropriately?, Cancer Treat. Rev. 37 (2011) 202-207. https://doi.org/10.1016/j.ctrv.2010.08.002.

[2] Y. Tabe, P.L. Lorenzi, M. Konopleva, Amino acid metabolism in hematologic malignancies and the era of targeted therapy, Blood. 134 (2019) 1014-1023. https://doi.org/10.1182/blood.2019001034.

[3] Glutaminase activity determines cytotoxicity of L-asparaginases on most leukemia cell lines, Leuk. Res. 39 (2015) 757-762. https://doi.org/10.1016/j.leukres.2015.04.008.

[4] T.N. Seyfried, G. Yu, J.C. Maroon, D.P.D. Agostino, Press-pulse $\square$ : a novel therapeutic strategy for the metabolic management of cancer, Nutr. Metab. (Lond). 14:19 (2017) 1-17. https://doi.org/10.1186/s12986-017-0178-2.

[5] H.A. Nguyen, Y. Su, A. Lavie, Design and characterization of erwinia chrysanthemi L-asparaginase variants with diminished L-glutaminase activity, J. Biol. Chem. 291 (2016) 17664-17676. https://doi.org/10.1074/jbc.M116.728485.

[6] R. Gribskov, A left-handed crossover involved in amidohydrolase structure catalysis of Erwinia chrysanthemi L-asparaginase with bound L-aspartate, 328 (1993) 275-279.

[7] Crystal structure of Escherichia coli L-asparaginase, an enzyme used in cancer therapy, Proc. Natl. Acad. Sci. U. S. A. 90 (1993) 1474-1478.

[8] N.E. Labrou, A.C. Papageorgiou, V.I. Avramis, Structure-Function Relationships Asparaginases and Clinical Applications of L- Asparaginases, Curr. Med. Chem. 17 (2010) 2183-2195.

[9] M. Maggi, S.D. Mittelman, J.H. Parmentier, G. Colombo, M. Meli, J.M. Whitmire, D.S. Merrell, J. Whitelegge, C. Scotti, A protease-resistant Escherichia coli asparaginase with outstanding stability and enhanced antileukaemic activity in vitro, Sci. Rep. 7 (2017) 1-16. https://doi.org/10.1038/s41598-017-15075-4.

[10] J. Aghaiypour, K; Wlodawer, A; Lubkowski, Structural Basis for the Activity and Substrate Specificity of, Biochemistry. 40 (2001) 5655-5664. https://doi.org/10.1074/jbc.M110.107177.

[11] Structural Insight into Substrate Selectivity of Erwinia chrysanthemi Lasparaginase, Biochemistry. 55 (2016) 1246-1253. https://doi.org/10.1021/acs.biochem.5b01351. 
[13] Development of L-Asparaginase Biobetters: Current Research Status and Review of the Desirable Quality Profiles, Front. Bioeng. Biotechnol. 6 (2019). https://doi.org/10.3389/fbioe.2018.00212.

[14] B. Asselin, C. Rizzari, Asparaginase pharmacokinetics and implications of therapeutic drug monitoring, Leuk. Lymphoma. 56 (2015) 2273-2280. https://doi.org/10.3109/10428194.2014.1003056.

790 [15] M.K. Schwartz, E.D. Lash, H.F. Oettgen, F.A. Tomao, L-asparaginase activity in plasma and other biological fluids, Cancer. 25 (1970) 244-252. https://doi.org/10.1002/1097-0142(197002)25:2<244::AIDCNCR2820250203>3.0.CO;2-V.

[16] J. Boos, G. Werber, E. Ahlke, P. Schulze-Westhoff, U. Nowak-Göttl, G. Würthwein, E.J. Verspohl, J. Ritter, H. Jürgens, Monitoring of asparaginase activity and asparagine levels in children on different asparaginase preparations, Eur. J. Cancer Part A. 32 (1996) 1544-1550. https://doi.org/10.1016/0959-8049(96)00131-1.

[17] Monitoring asparaginase activity in middle-income countries, Lancet. Oncol. 19 (2018) 1149-1150. https://doi.org/10.1016/S1470-2045(18)30584-9.

[18] Low Bioavailability and High Immunogenicity of a New Brand of E. coli IAsparaginase with Active Host Contaminating Proteins, EBioMedicine. 30 (2018) 158-166. https://doi.org/10.1016/j.ebiom.2018.03.005.

[19] M.K. Parr, O. Montacir, H. Montacir, Physicochemical characterization of biopharmaceuticals, J. Pharm. Biomed. Anal. 130 (2016) 366-389. https://doi.org/10.1016/j.jpba.2016.05.028.

[20] mMass data miner: an open source alternative for mass spectrometric data analysis, Rapid Commun. Mass Spectrom. RCM. 22 (2008) 905-908. https://doi.org/10.1002/rcm.3444.

810 [21] L.C. Palmieri, B. Melo-ferreira, C.A. Braga, G.N. Fontes, L.J. Mattos, L.M.T.R. Lima, Stepwise oligomerization ofmurine amylin and assembly ofamyloid fibrils., Biophys. Chem. 180-181 (2013) 135-144. https://doi.org/10.1016/j.bpc.2013.07.013.

[22] Use of ion mobility mass spectrometry and a collision cross-section algorithm to study an organometallic ruthenium anticancer complex and its adducts with a DNA oligonucleotide, Rapid Commun. Mass Spectrom. RCM. 23 (2009) 3563-3569. https://doi.org/10.1002/rcm.4285.

[23] W. Kabsch, research papers XDS research papers, Acta Crystallogr. Sect. D 
Biol. Crystallogr. D66 (2010) 125-132. https://doi.org/10.1107/S0907444909047337.

[24] Coot: model-building tools for molecular graphics, Acta Crystallogr. Sect. D Biol. Crystallogr. 60 (2004) 2126-2132. https://doi.org/10.1107/S0907444904019158.

[25] REFMAC5 for the refinement of macromolecular crystal structures, Acta Crystallogr. Sect. D Biol. Crystallogr. 67 (2011) 355-367.
https://doi.org/10.1107/S0907444911001314.

[26] The Phenix Software for Automated Determination of Macromolecular Structures, Methods. 55 (2011) 94. https://doi.org/10.1016/j.ymeth.2011.07.005.

830 [27] A. Viegas, J. Manso, F.L. Nobrega, E.J. Cabrita, Saturation-transfer difference (STD) NMR: A simple and fast method for ligand screening and characterization of protein binding, J. Chem. Educ. 88 (2011) 990-994. https://doi.org/10.1021/ed101169t.

[28] S.M. Kelly, T.J. Jess, N.C. Price, How to study proteins by circular dichroism, 1751 (2005) 119-139. https://doi.org/10.1016/j.bbapap.2005.06.005.

[29] The Small-Angle X-ray Scattering Beamline of the Brazilian Synchrotron Light Laboratory, J. Appl. Crystallogr. 30 (1997) 880-883. https://doi.org/10.1107/S0021889897001829.

[30] FIT2D: a multi-purpose data reduction, analysis and visualization program, J. Appl. Crystallogr. 49 (2016) 646-652. https://doi.org/10.1107/S1600576716000455.

[31] ATSAS 2.8: a comprehensive data analysis suite for small-angle scattering from macromolecular solutions, J. Appl. Crystallogr. 50 (2017) 1212-1225. https://doi.org/10.1107/S1600576717007786.

845 [32] P. V Konarev, V. V Volkov, A. V Sokolova, H.J. Koch, D.I. Svergun, PRIMUS $\square$ : a Windows PC-based system for small- angle scattering data analysis, J. Appl. Cryst. 36 (2003) 1277-1282.

[33] B.T. Ruotolo, J.L.P. Benesch, A.M. Sandercock, S. Hyung, C. V Robinson, Ion mobility - mass spectrometry analysis of large protein complexes, 3 (2008) 1139-1152. https://doi.org/10.1038/nprot.2008.78.

[34] R. Salbo, M.F. Bush, H. Naver, I. Campuzano, C. V Robinson, I. Pettersson, T.J.D. Jørgensen, K.F. Haselmann, Traveling-wave ion mobility mass spectrometry of protein complexes $\square$ : accurate calibrated collision crosssections of human insulin oligomers, Rapid Commun. Mass Spectrom. 26 (2012) 1181-1193. https://doi.org/10.1002/rcm.6211. 
[35] C. Derst, A. Wehner, V. Specht, K. -H Röhm, States and Functions of Tyrosine Residues in Escherichia coli Asparaginase II, Eur. J. Biochem. 224 (1994) 533-540. https://doi.org/10.1111/j.1432-1033.1994.00533.x.

[36] N. Nakamura, Y. Morikawa, M. Tanaka, L-asparaginase from escherichia coli: Part II. Substrate specificity studies, Agric. Biol. Chem. 35 (1971) 743751. https://doi.org/10.1080/00021369.1971.10859979.

[37] M. Sanches, S. Krauchenco, I. Polikarpov, Structure, Substrate Complexation and Reaction Mechanism of Bacterial Asparaginases, Curr. Chem. Biol. 1 (2012) 75-86. https://doi.org/10.2174/2212796810701010075.

865 [38] A dyad of lymphoblastic lysosomal cysteine proteases degrades the antileukemic drug L-asparaginase, J. Clin. Invest. 119 (2009) 1964-1973. https://doi.org/10.1172/JCI37977.

[39] S.F. Altschul, T.L. Madden, A.A. Schäffer, J. Zhang, Z. Zhang, W. Miller, D.J. Lipman, Gapped BLAST and PSI-BLAST $\square$ : a new generation of protein database search programs, 25 (1997) 3389-3402.

[40] S.F. Altschul, J.C. Wootton, E.M. Gertz, R. Agarwala, A. Morgulis, A.A. Scha, Protein database searches using compositionally adjusted substitution matrices, 272 (2005) 5101-5109. https://doi.org/10.1111/j.17424658.2005.04945.x.

[41] Engineering the substrate specificity of Escherichia coli asparaginase. II. Selective reduction of glutaminase activity by amino acid replacements at position 248, Protein Sci. A Publ. Protein Soc. 9 (2000) 2009-2017. https://doi.org/10.1110/ps.9.10.2009.

[42] A.P. Sudhir, B.R. Dave, A.S. Prajapati, K. Panchal, D. Patel, R.B. Subramanian, Characterization of a Recombinant Glutaminase-Free IAsparaginase (ansA3) Enzyme with High Catalytic Activity from Bacillus licheniformis, Appl. Biochem. Biotechnol. 174 (2014) 2504-2515. https://doi.org/10.1007/s12010-014-1200-z.

[43] A. Beckett, D. Gervais, What makes a good new therapeutic I ? asparaginase $\square$ ?, World J. Microbiol. Biotechnol. 9 (2019) 1-13. https://doi.org/10.1007/s11274-019-2731-9.

[44] J.J.M. Cachumba, F.A.F. Antunes, G.F.D. Peres, L.P. Brumano, J.C. Dos Santos, S.S. Da Silva, Current applications and different approaches for microbial L-asparaginase production, Brazilian J. Microbiol. 47 (2016) 77-85. https://doi.org/10.1016/j.bjm.2016.10.004.

[45] L.M.T.R. Lima, T.S. Araujo, F.C.L. Almeida, M.S. Almeida, Monitoring, Lancet Oncol. 19 (2018) e574. https://doi.org/10.1016/S14702045(18)30656-9. 
[46] Structures of two highly homologous bacterial l--asparaginases: a case of enantiomorphic space groups, Acta Crystallogr. Sect. D Biol. Crystallogr. 57 (2001) 369-377. https://doi.org/10.1107/S0907444900020175.

[47] X. Yu, Y. Wu, F. Huang, J. Ulrich, J. Wang, Purification of Recombinant Lasparaginase II Using Solvent Freeze Out ( SFO ) Technology, Chem. Eng. Technol. 41 (2018) 1080-1085. https://doi.org/10.1002/ceat.201700569.

900 [48] Office TS 2017, Pharmacopoeia of the People's Republic of China 2015., in: B, Beijing: The Stationery Office., n.d.

[49] R.A. Egler, S.P. Ahuja, Y. Matloub, L-asparaginase in the treatment of patients with acute lymphoblastic leukemia, J. Pharmacol. Pharmacother. 7 (2016) 62-71. https://doi.org/10.4103/0976-500X.184769.

905 [50] M. Mohseni, H. Uludag, J.M. Brandwein, Advances in biology of acute lymphoblastic leukemia (ALL) and therapeutic implications., Am. J. Blood Res. 8 (2018) 29-56.

[51] C. Lanvers-Kaminsky, Asparaginase pharmacology: Challenges still to be faced, Cancer Chemother. Pharmacol. 79 (2017) 439-450. https://doi.org/10.1007/s00280-016-3236-y. 


\section{Figures}

A

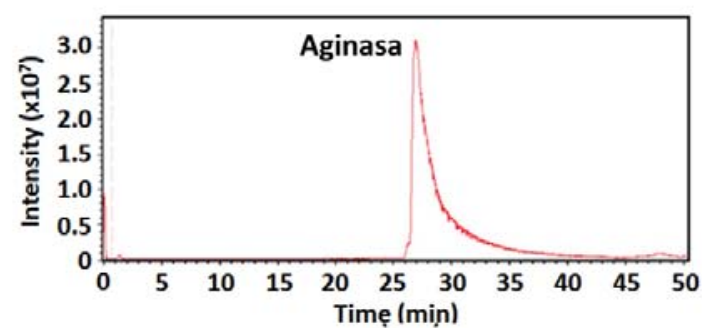

C

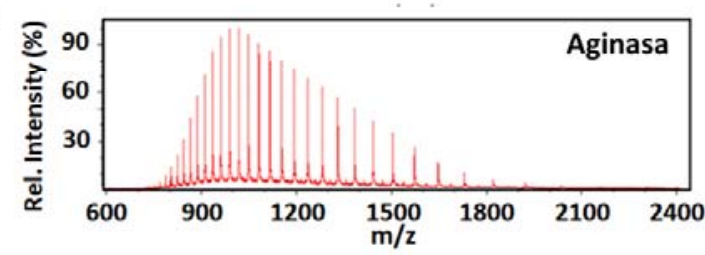

D

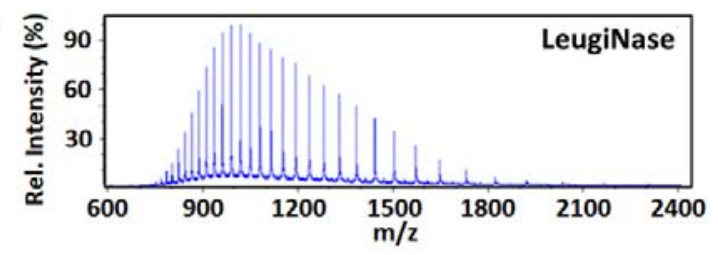

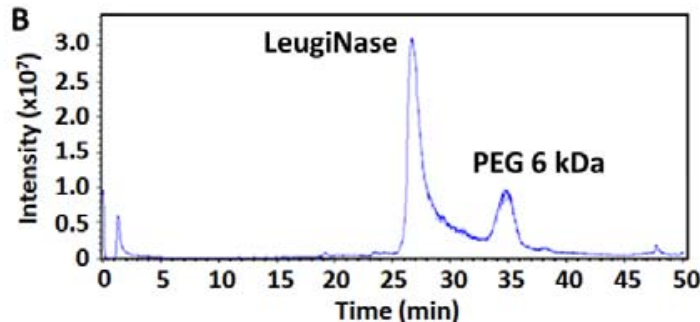

E

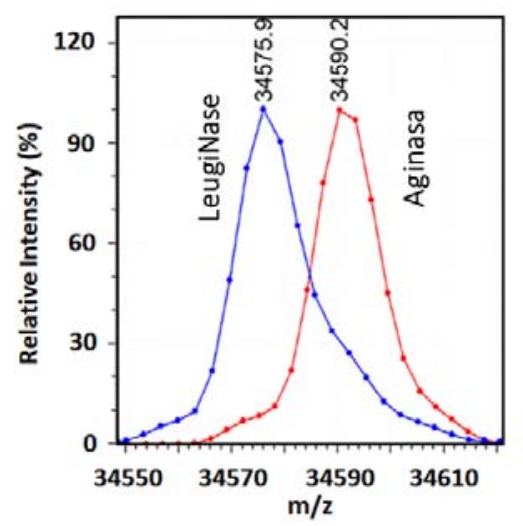

Figure 1. Characterization of asparaginase products by LC-ESI-MS. The EcA2 products Aginasa and Leuginase were evaluated by LC-ESI-MS. In the total ion current chromatograms, A. Aginasa and B. Leuginase showed a major peak corresponding to the respective intact proteins, and a peak corresponding to PEG with $6 \mathrm{kDa}$ was found in Leuginase (Fig. S2). Charge envelopes C. and D. reveal the prevalence of monomers and minor contributions of dimers (smaller peaks with a thicker line between charged states belonging to monomers). E. The deconvoluted spectra revealed a mass of 34,590.2 Da for Aginasa and 34,575.9 Da for Leuginase. 

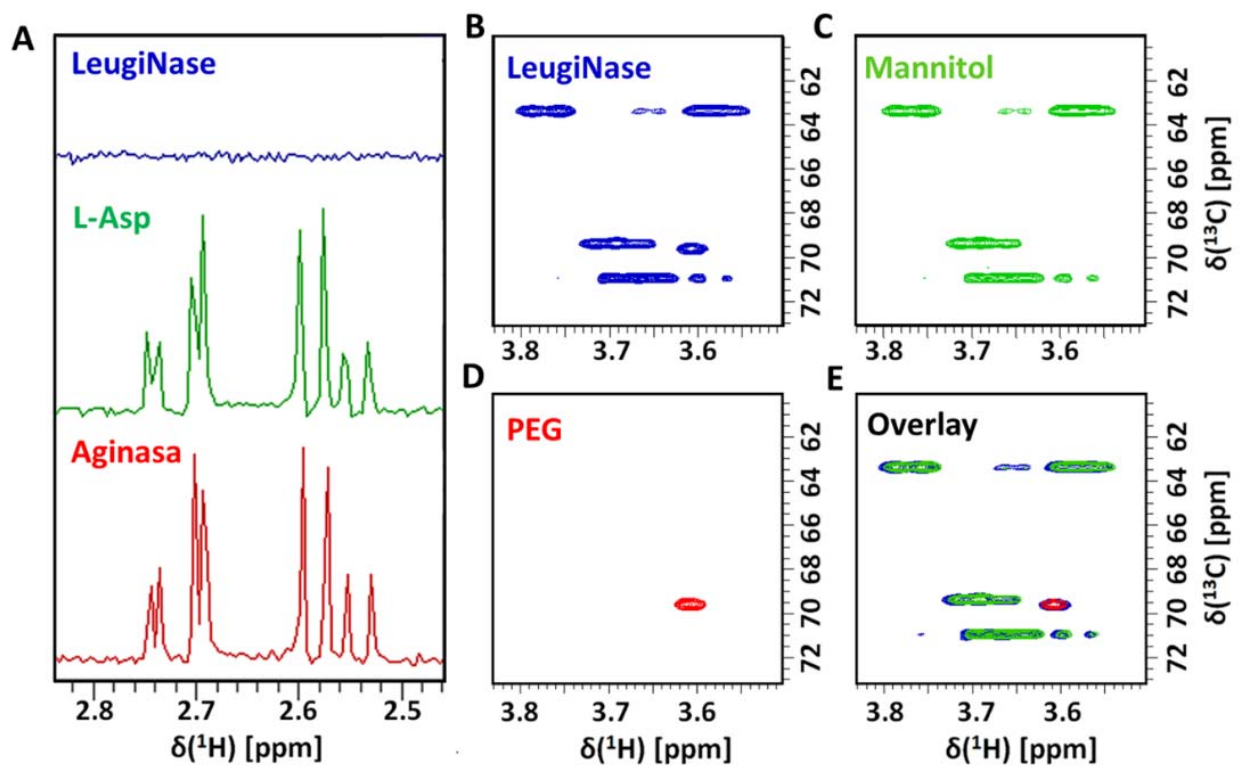

Figure 2. Identification of the constituents of EcA2 preparations using NMR A. 1D ${ }^{1} \mathrm{H}$ NMR spectra of L-Asp (green) and ultrafiltrated EcA2 formulations, Aginasa (red) and Leuginase (blue). Spectra were collected in a $400 \mathrm{MHz}$ spectrometer at $298 \mathrm{~K} .2 \mathrm{D}\left[{ }^{1} \mathrm{H},{ }^{13} \mathrm{C}\right]-\mathrm{HSQC}$ spectrum of $\mathbf{B}$. Leuginase total formulation, and standard solutions of C. mannitol and D. PEG. E. Overlay of the 2D spectra supports the presence of these constituents in the Leuginase formulation.
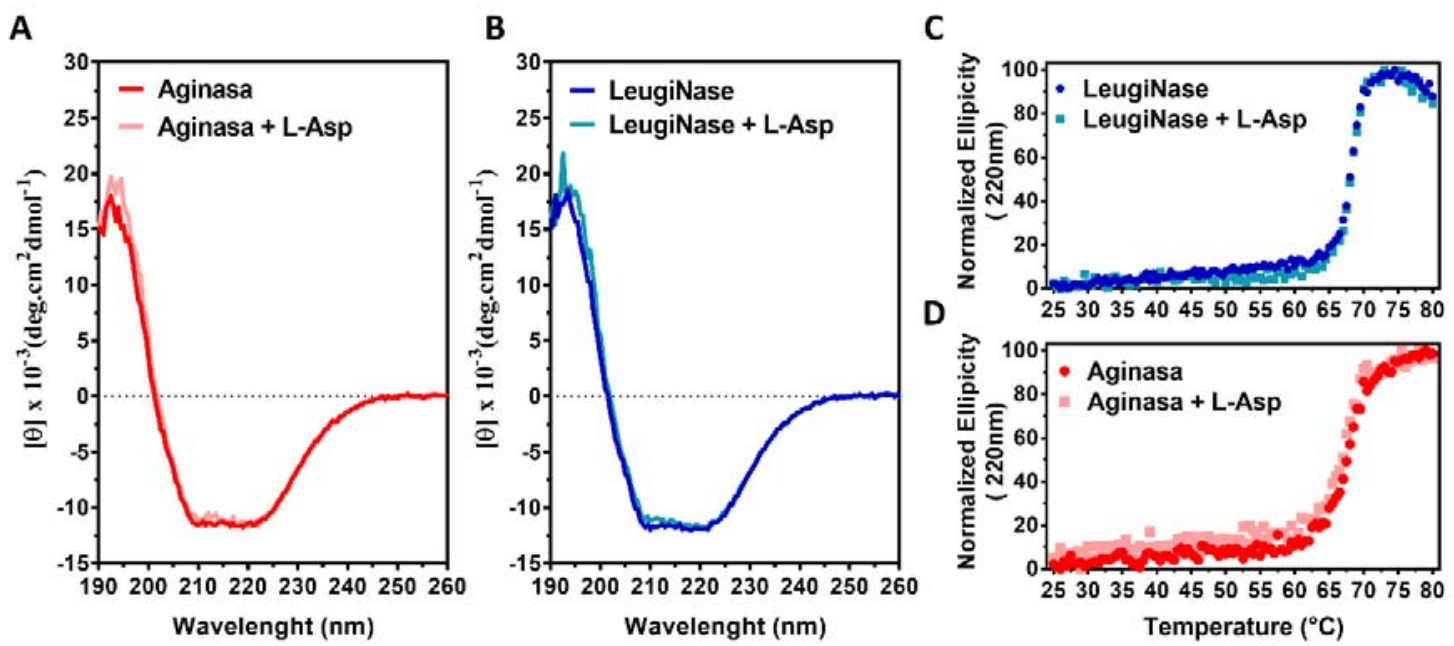

Figure 3. Thermal stability of EcA2. We monitored the thermal stability of EcA2 products, Aginasa and Leuginase, using circular dichroism (CD). CD spectra of A. Aginasa and B. Leuginase in the absence or presence of L-Asp showing only minor changes in absolute intensity. We tested the thermal stability of both C. Aginasa and D. Leuginase in the absence or presence of L-Asp. Sigmoidal fitting from $25^{\circ} \mathrm{C}$ to $80^{\circ} \mathrm{C}$ allowed the recovery of their transition midpoint and slope (shown in Table S1). 

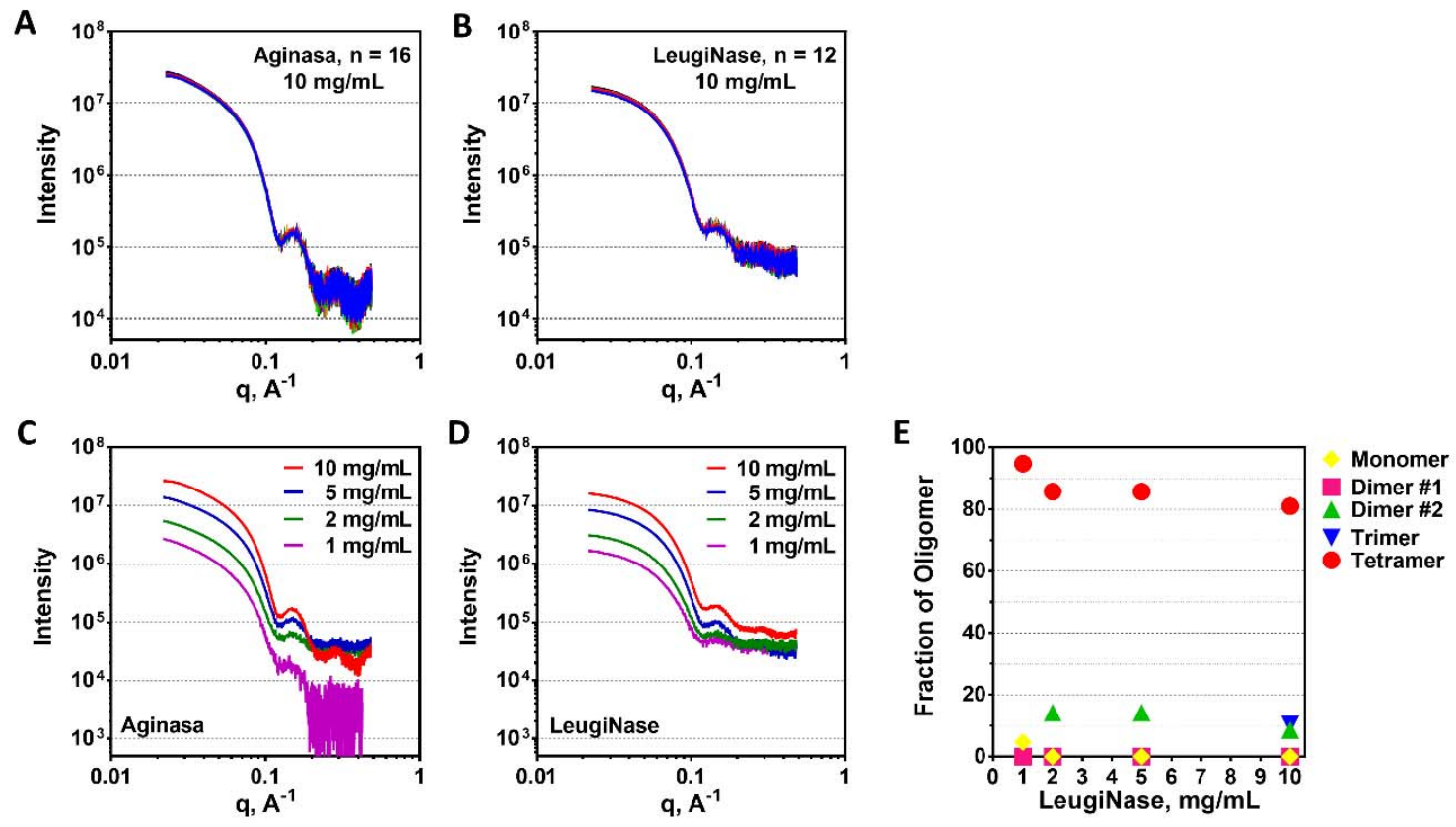

Figure 4. Small-angle X-ray scattering profile of EcA2 formulations. Each curve represents one sample at a different time from resuspension of the lyophilized product to data collection for $\mathbf{A}$. Aginasa and B. Leuginase. Samples were stored at $4^{\circ} \mathrm{C}$ until measurements were performed at $25^{\circ} \mathrm{C}$. Scattering profile of C. Aginasa and D. Leuginase at different protein concentrations. E. The fractions of monomer and the oligomers of Leuginase is shown for each concentration used.
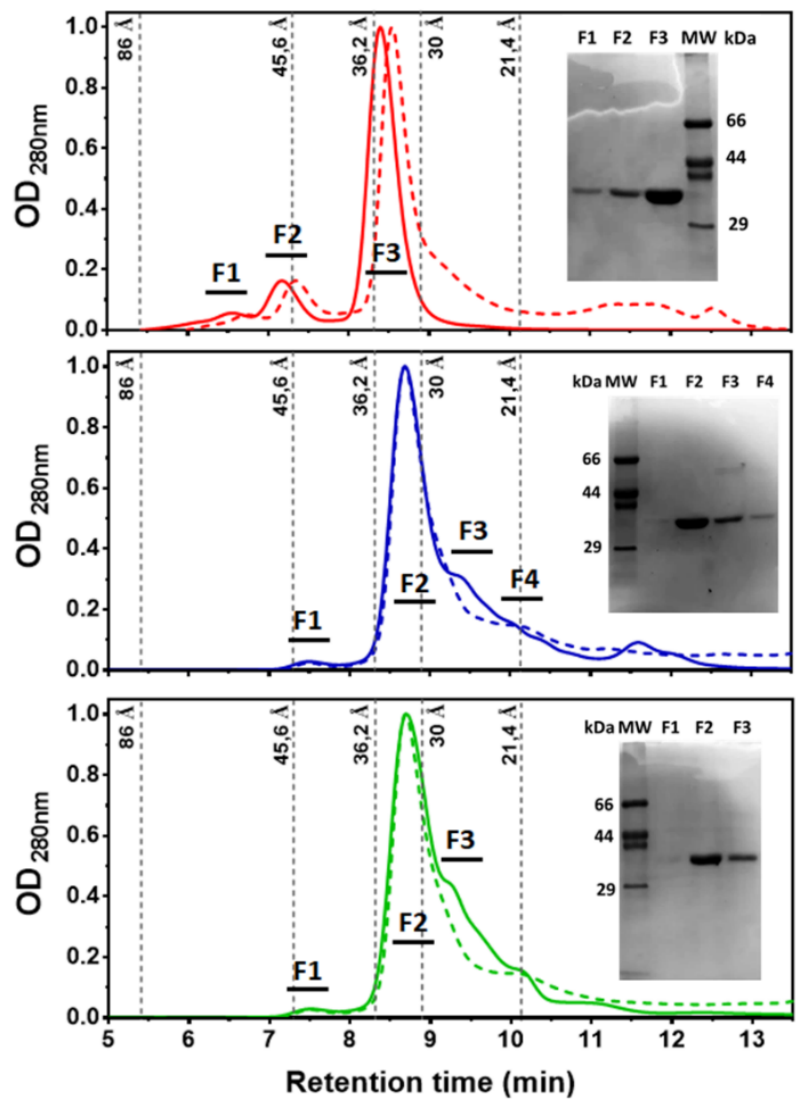
Figure 5. Size-exclusion chromatography analysis of Aginasa (red), Leuginase (blue), and Leuginase supplemented with $1 \mathrm{mM}$ L-Asp (green). Analysis by SDS-PAGE $15 \%$ of the fractions obtained for each peak, confirming EcA2 elution. The hydrodynamic radius of standard globular proteins is indicated at their retention volume. Chromatograms with solid lines represent the sample at $320 \mu \mathrm{M}$, while dashed lines are for samples diluted to $10 \mu \mathrm{M}$. OD $280 \mathrm{~nm}$ was normalized to facilitate comparison of chromatographic runs.
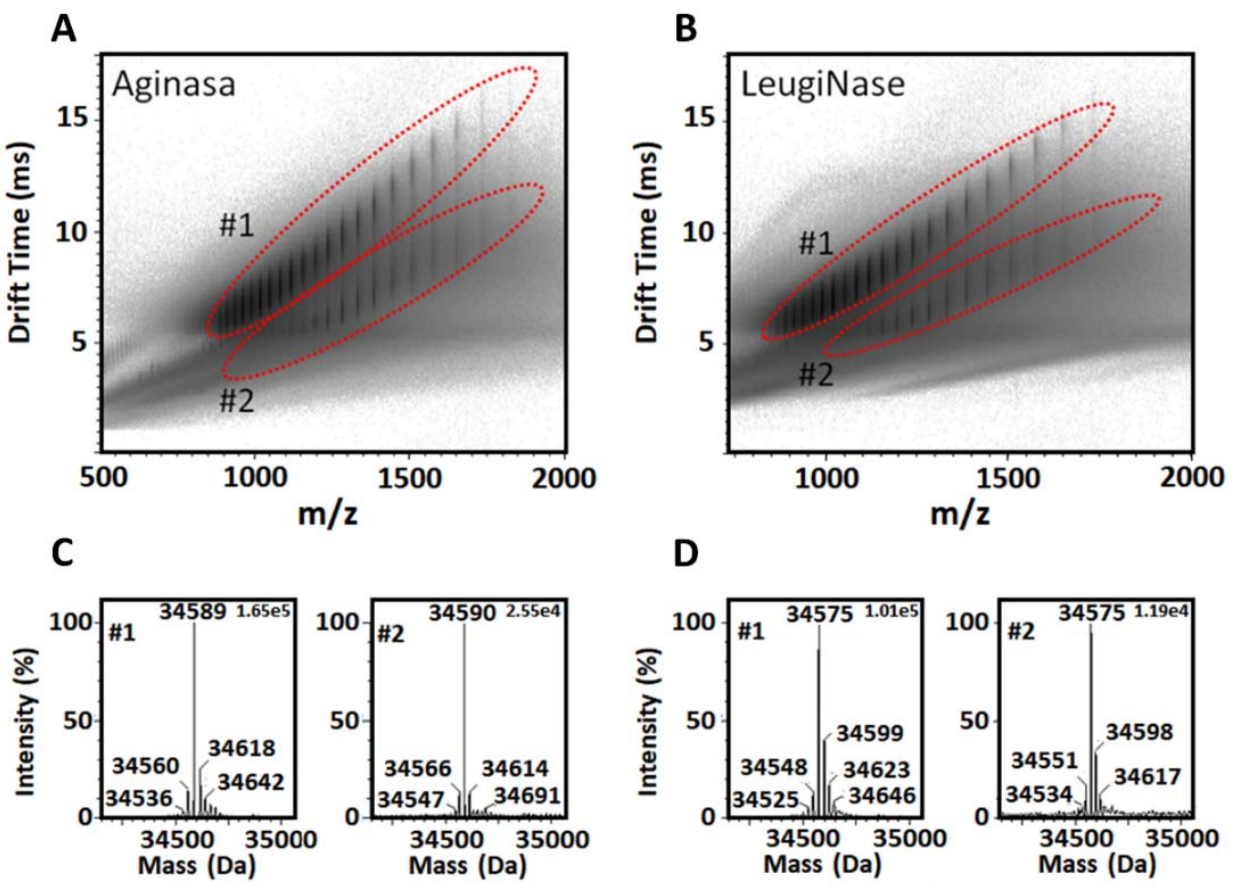

960 Figure 6. Multiple monomeric conformers of L-asparaginase as revealed by ion mobility spectrometry - mass spectrometry. A. Aginasa and B. Leuginase evaluated at $1 \mu \mathrm{M}$ by ESI-IMSMS in $0.1 \%$ formic acid, showing two charge species envelopes (dotted red ellipses), corresponding to conformers with same molecular mass as shown by their deconvoluted spectra (C and D, respectively) using MaxEnt1 (MassLynx, Waters). Measurements performed in Synapt G1

965 (Waters) by direct injection. 

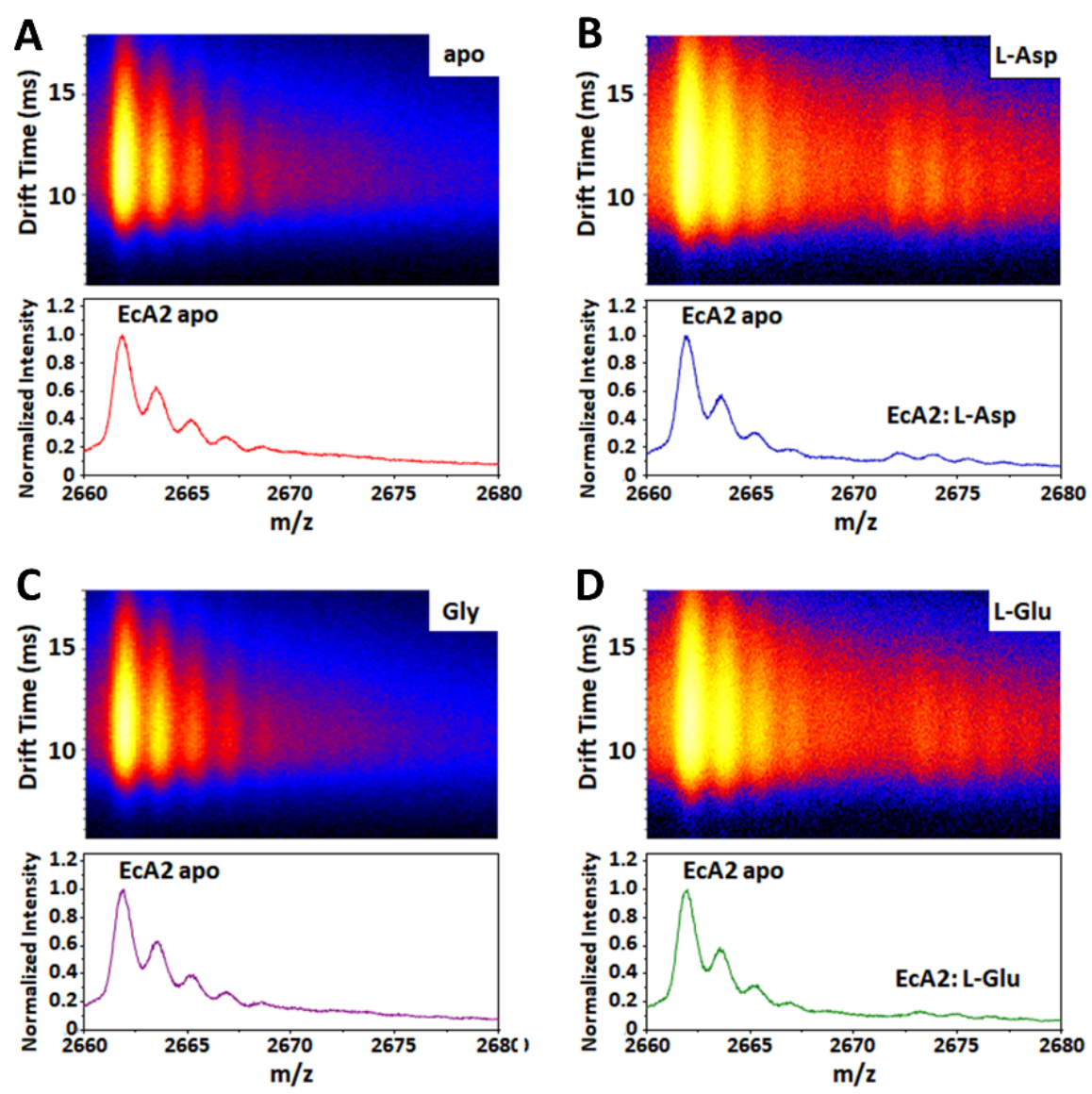

970 Figure 7. Mapping interaction of amino acid with EcA2 by mass spectrometry. The ESI-IMS-MS spectra of EcA2 were measured $\mathbf{A}$. in the absence or the presence of $\mathbf{B}$. L-aspartic acid, C. glycine, or D.L-glutamic acid. Only the M+13 charged state is shown for each sample, revealing the peaks corresponding to the free and the amino acid-bound forms of the protein and salt adducts (most likely $\mathrm{Na}$ ). Other charged states are illustrated in Fig. S8, indicating similar results. 
A

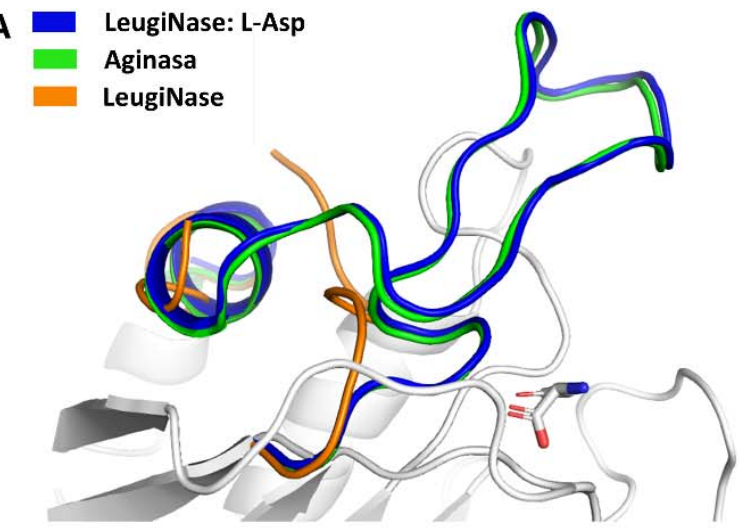

B

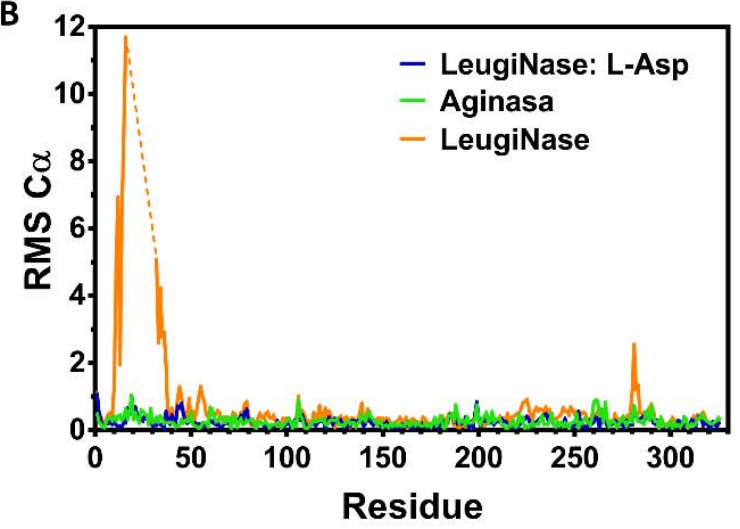

Figure 8. Crystallographic analysis of EcA2. Aginasa and Leuginase were subjected to crystallization and x-ray diffraction, and the structure was solved by molecular replacement. Aginasa (PDB Id.: 6UOH) showed two monomers in the asymmetric unit (A.U.) with bound L-Asp in the closed active site. In comparison, apo Leuginase (PDB Id.: 6UOD) showed four monomers in the A.U. and open active site with undefined lid. The co-crystallized Leuginase: L-Asp (PDB Id.: 6UOG) resulted in eight monomers in the A.U. with a closed lid over the amino acid-bound active site. A. Detailed view of the active-site lid, indicating the superposition between Asp-Aginasa and Asp-Leuginase. B. RMSD plot of the superposition of the crystal structures obtained, demonstrating the similarity in the spatial distribution along with the protein, except for the active-site lid (Residues G10 to N37) of the apo form of Leuginase. Data collection and refinement statistics can be found in the Table $\mathrm{S} 3$.
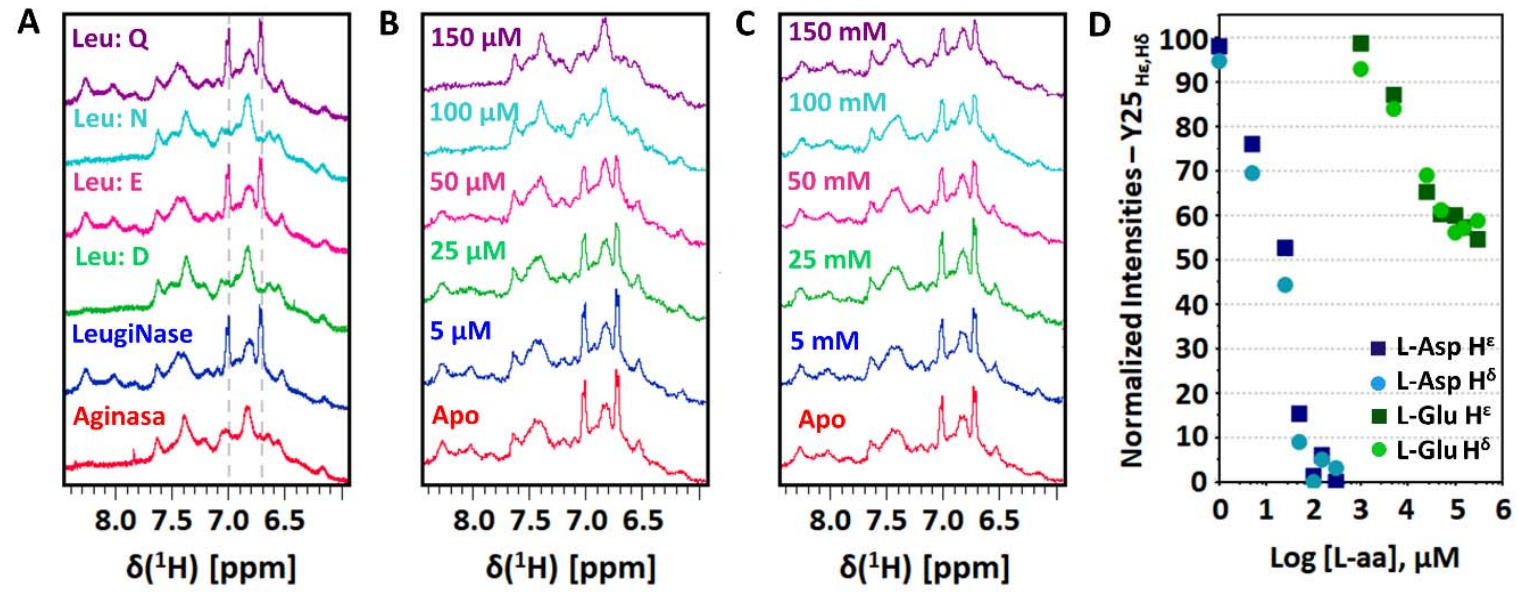

Figure 9. Monitoring Y25 dynamics upon L-aa binding by NMR. A. Multiple displays of $1 \mathrm{D}{ }^{1} \mathrm{H}$ NMR spectra of Aginasa and Leuginase whole formulation $(320 \mu \mathrm{M})$ and Leuginase added with 1 $\mathrm{mM}$ of different L-amino acids indicated with the one-letter code above each spectrum. For each experiment, a total of 512 scans with $2 \mathrm{~s}$ of relaxation delay were collected in a $400 \mathrm{MHz}$ spectrometer at $298 \mathrm{~K}$. In the presence of L-Asp and L-Asn, the Tyr25 pair of sharp signals attributed to $\mathrm{H}^{\varepsilon}$ and $\mathrm{H}^{\delta}$ (marked with a dotted gray line) disappear, indicating a loss of flexibility. Multiple displays of NMR-titration analysis of $160 \mu \mathrm{M}$ Leuginase supplemented with increasing concentrations of B. L-Asp or C. L-Glu. Concentration of L-aa used for the acquisition of each spectrum is indicated in the figure. For each experiment, a total of 256 scans with $2 \mathrm{~s}$ of relaxation delay were collected in a $400 \mathrm{MHz}$ spectrometer at $298 \mathrm{~K}$. D. The quantitative analysis of the NMRtitration. 

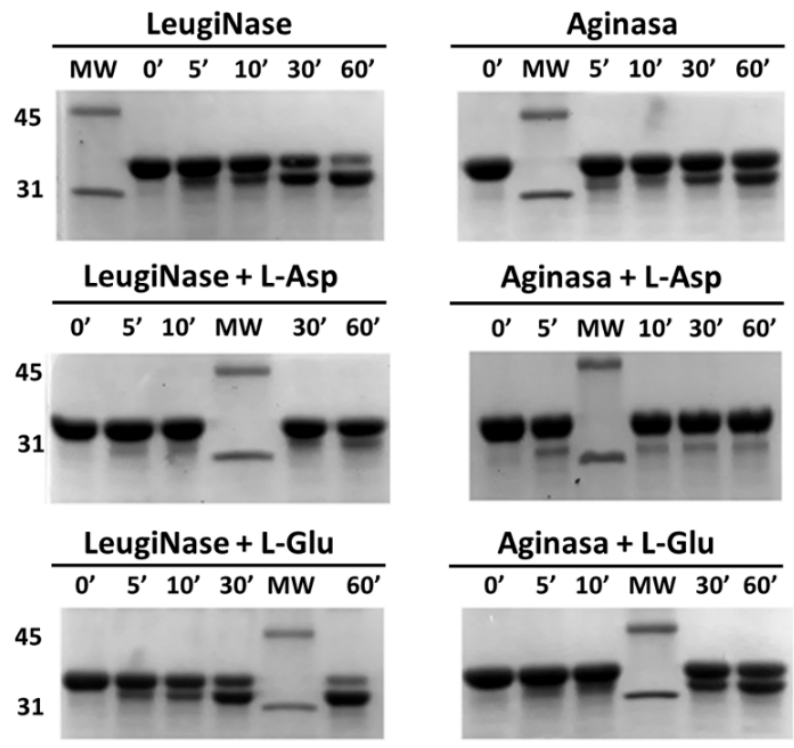

1010 Figure 10. L-Asp protects EcA2 against limited proteolysis. SDS-PAGE $15 \%$ analysis of Aginasa and Leuginase $(280 \mu \mathrm{M})$, with and without L-Asp or L-Glu addition $(2.8 \mathrm{mM})$, after incubation with Proteinase $\mathrm{K}$ among indicated time. Two bands of the molecular weight (MW) ladder are indicated on the left ( 31 and $45 \mathrm{kDa})$.

A

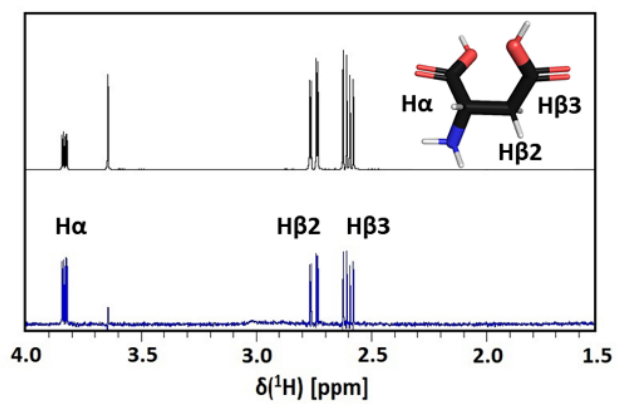

B

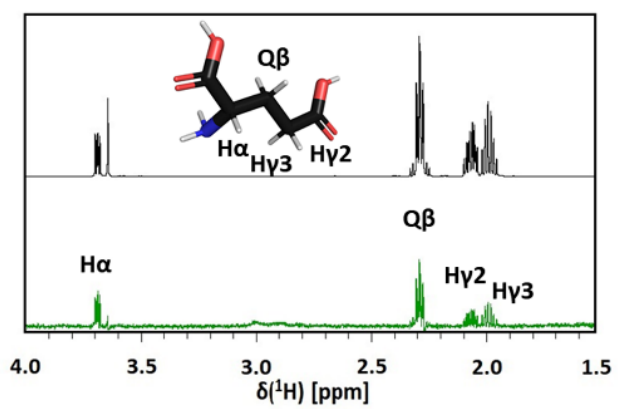

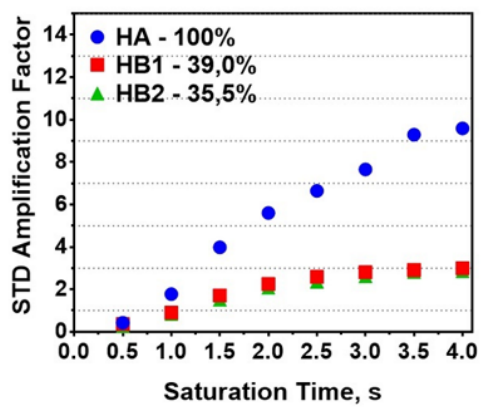

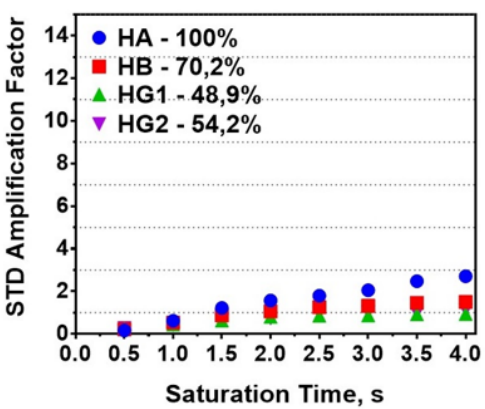

1015 Figure 11. STD-NMR analysis reveal differences in substrate recognition of EcA2 for different ligands. The reference spectrum acquired for each ligand is in black, and the corresponding STDNMR spectrum is colored in blue for A. L-Asp and green for B. L-Glu. The chemical structure of amino acids, BMRB entry bmse000031 (L-Asp) and bmse000037 (L-Glu), with assigned protons presented in each spectrum. The STD amplification factor as a function of saturation time is represented alongside each spectrum with relative STD percentages indicated for each assigned proton. 


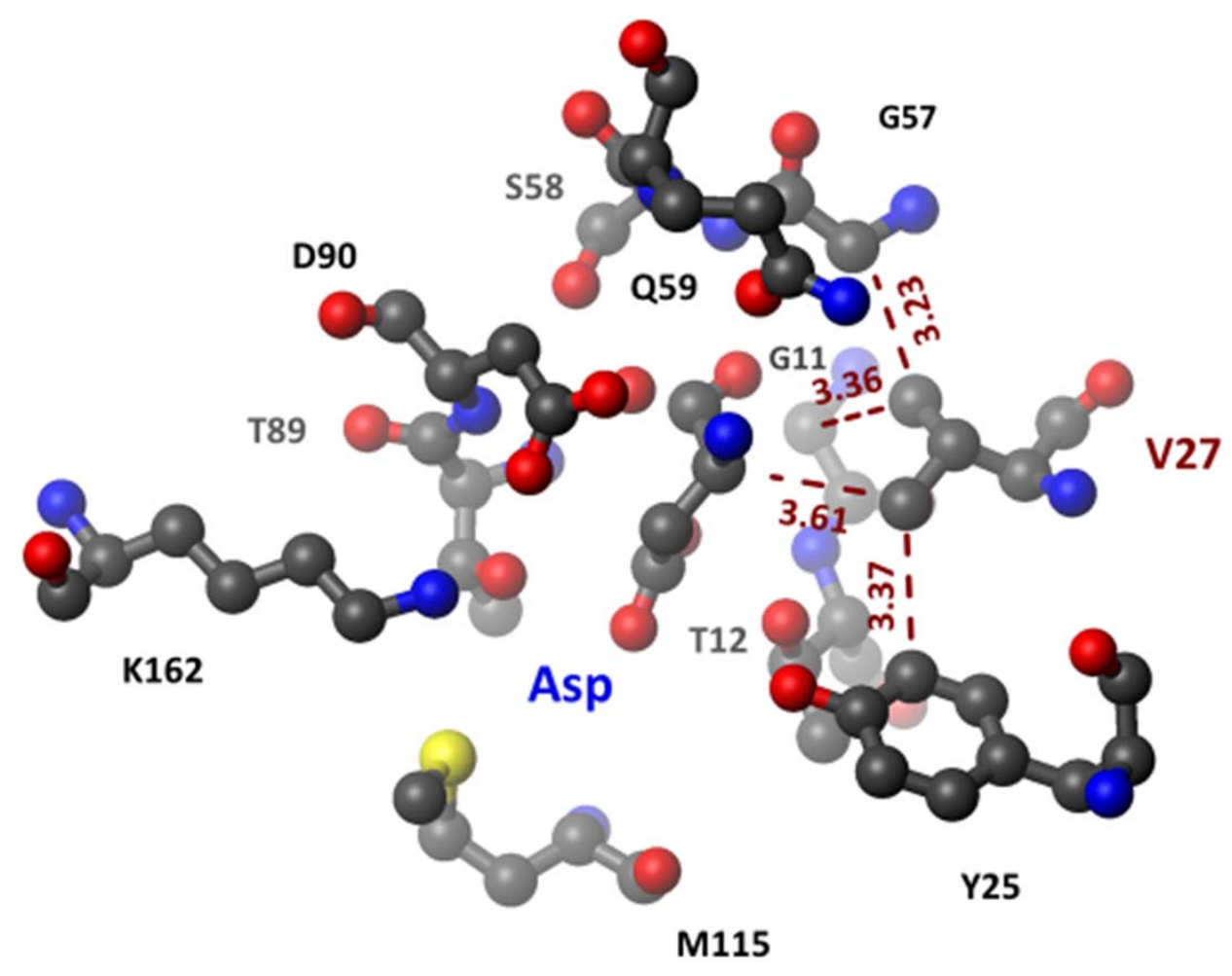

1025 Figure 12. Stereochemical analysis of EcA2 catalytic site upon L-Asp binding. The figure illustrates the ball and stick representation of the EcA2 catalytic site (Leuginase: Asp - PDB Id.: 6UOG) pointing out Val27 interactions (represented by red dashed lines) together with the distances in Angstroms estimated between carbon atoms. All the estimated distances between Val27 and the catalytic site residues and the ligand L-Asp can be found in Table S5 and S6. Despite ligand binding 1030 being maintained mostly by hydrogen bonds (LigPlot shown in Fig. S11), Val27 appears to play a role linking the ligand binding with conformational change toward the active closed lid form. As demonstrated in the figure, Val27 presents a network of hydrophobic interactions, with the ligand L-Asp $\left(\mathrm{H}^{\alpha}\right)$, active site residues G57 and G11, and the sidechain of the loop residue Y25. 


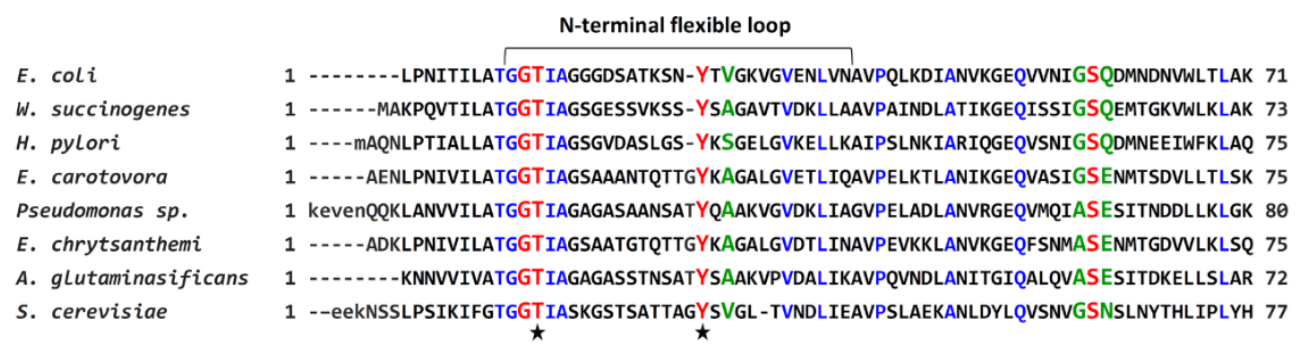

E. coli

W. succinogenes

H. pylori

E. carotovora

Pseudomonas sp.

E. chrysanthemi

A. gLutaminasificans

S. cerevisiae

E. coli

W. succinogenes

H. pylori

E. carotovora

Pseudomonas sp.

E. chrytsanthemi

A. glutaminasificans

S. cerevisiae

E. coli

W. succinogenes

H. pylori

E. carotovora

Pseudomonas sp.

E. chrytsanthemi

A. glutaminasificans

S. cerevisiae

E. coli

W. succinogenes

H. pylori

E. carotovora

Pseudomonas sp

E. chrytsanthemi

A. glutaminasificans

s. cerevisiae
72 KINTDC--DKTD- -GFVITHGTDTMEETAYFLDLTVKCDKPVVMVGAMRPSTSMSADGPFNLYNAVVTAADKASANRGVL 147 74 RVNELLAQKETE - -AVIITHGTDTMEETAFFLNLTVKSQKPVVLVGAMRSGSSMSADGPMNLYNAVNVAINKASTNKGVV 151 76 RAQELLDDSRIQ--GVVITHGTDTLEESAYFLNLVLHSTKPVVLVGAMRNASSLSADGALNLYEAVSVAVNEKSANKGVL 153 76 RVNELLARSDVD--GVVITHGTDTLDESPYFLNLTVKSDKPVVFVAAMRPATAISADGPMNLYGAVKVAADKNSRGRGVL 153 81 RVAELADSNDVD--GIVITHGTDTLEETAYFLDLTLNTDKPIVVVGSMRPGTAMSADGMLNLYNAVAVASNKDSRGKGVL 158 76 RVNELLARDDVD- -GVVITHGTDTVEESAYFLHLTVKSDKPVVFVAAMRPATAISADGPMNLLEAVRVAGDKQSRGRGVM 153 73 QVNDLVKKPSVN--GVVITHGTDTMEETAFFLNLVVHTDKPIVLVGSMRPSTALSADGPLNLYSAVALASSNEAKNKGVM 150 78 GISEALASD--DYaGAVVTHGTDTMEETAFFLDLTINSEKPVCIAGAMRPATATSADGPMNLYQAVSIAASEKSLGRGTM 155 $\star \star$

Figure 13. Sequence alignment of L-asparaginases. Conserved residues are colored in blue. Conserved active site residues are highlighted in red, with those described as being directly involved in catalysis indicated by black stars. Residues within the catalytic site that are not well1045 conserved are shown in green. Residue E283, which shows high C $\alpha$ RMS in Leuginase Apo structure (Figure $8 \mathrm{~B}$ ), is highlighted in orange. This difference is due to its role in ligand binding when the residue is in the adjacent chain that forms the intimate dimer (Fig. S11). The flexible Nterminal loop boundaries of EcA2, identified through the $\mathrm{C} \alpha$ RMS displacement analysis, are indicated in the figure. The UniProt entries of the sequences used in this alignment, with identities 1050 (I) and homology (H) compared to EcA2 indicated in parenthesis, were: Escherichia coli P00805, Wolinella succinogenes (I - 55\%; H - 72\%) P50286, Helicobacter pylori (I - 50\%; H - 69\%) Q9ZLB9, Erwinia carotovora (I - 49\%; H - 65\%) Q7WWK9, Pseudomonas sp. (I - 47\%; H 67\%) P10182, Erwinia chrysanthemi P06608 (I - 48\%; H - 63\%), Acinetobacter glutaminasificans 
(I - 45\%; H - 64\%) P10172, and Saccharomyces cerevisiae (I - 41\%; H - 56\%) P0CX77. The

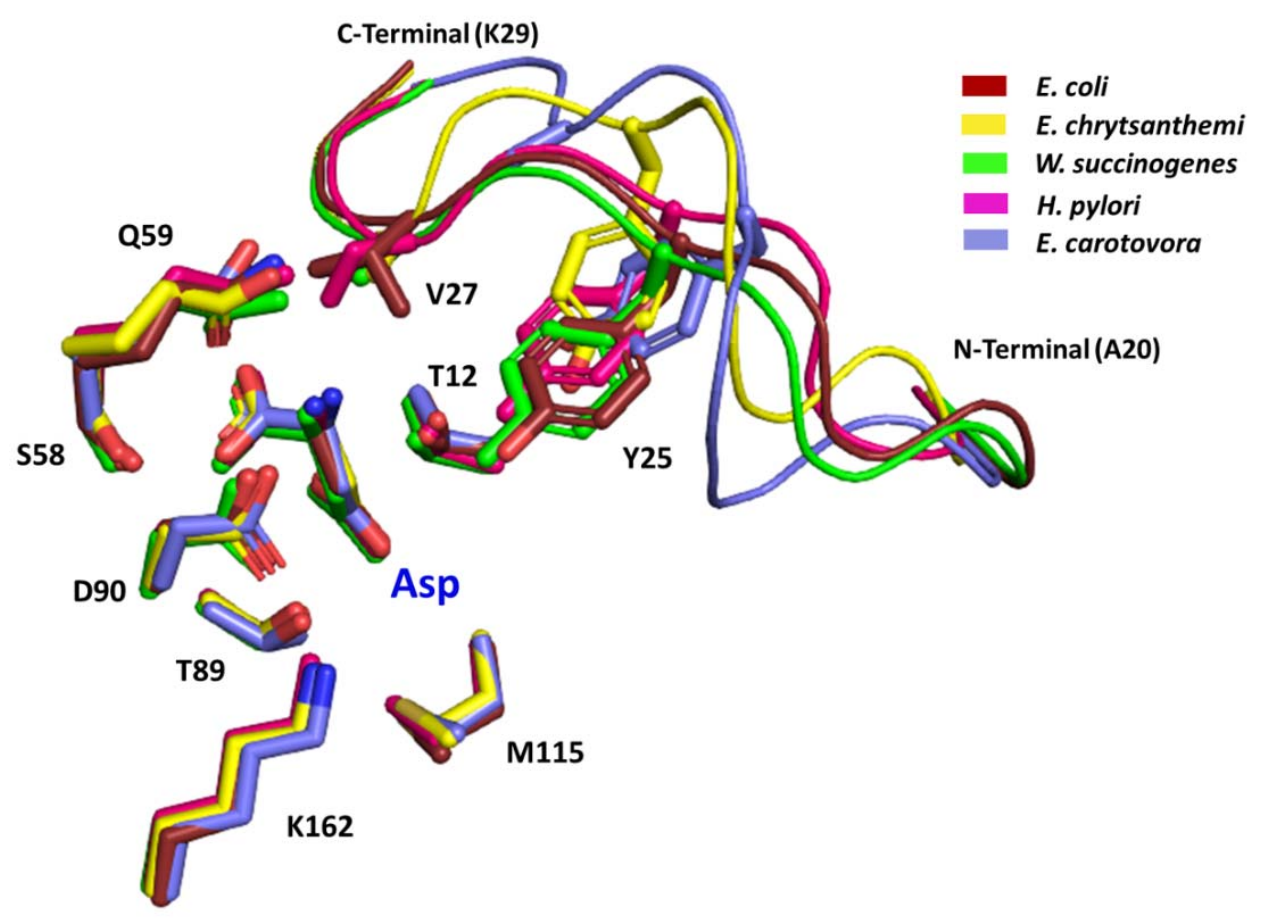

Figure 14. Superposition of the L-asparaginase catalytic site. The structural alignment demonstrates that L-asparaginases from different species exhibit high conservation of catalytic site structural features with a major difference located at the loop forming the lid coordinates, specially Val27 and Tyr25, which are crucial for loop stabilization. Only L-asparaginases with structures solved with LAsp were superposed. Residues are labeled according to the EcA2 sequence. The PDB entries of corresponding structures used for this alignment were Wolinella succinogenes PDB ID 5K3O, 1065 Escherichia coli (Leuginase-Asp) PDB ID 6UOG, Erwinia chrysanthemi PDB ID 5F52, Erwinia carotovora PDB ID 2GVN, Helicobacter pylori PDB ID 2WLT. 